\title{
Braess' paradox in the age of traffic information
}

\author{
S Bittihn and A Schadschneider \\ Institut für Theoretische Physik, Universit ät zu Köln, 50937 Köln, Germany \\ E-mail: as@thp.uni-koeln.de, bittihn@thp.uni-koeln.de
}

\begin{abstract}
The Braess paradox describes the counterintuitive situation that the addition of new roads to road networks can lead to higher travel times for all network users. Recently we could show that user optima leading to the paradox exist in networks of microscopic transport models. We derived phase diagrams for two kinds of route choice strategies that were externally tuned and applied by all network users. Here we address the question whether these user optima are still realized if intelligent route choice decisions are made based upon two kinds of traffic information. We find that the paradox still can occur if the drivers 1) make informed decisions based on their own past experiences or 2) use traffic information similar to that provided by modern navigation apps. This indicates that modern traffic information systems are not able to resolve Braess' paradox.
\end{abstract}

Keywords: traffic, network, Braess' paradox, exclusion process

Submitted to: J. Stat. Mech.

7 September 2020 


\section{Contents}

1 Introduction $\quad 2$

2 Background information

2.1 Some terminology . . . . . . . . . . . . . . . . . 5

2.2 The Braess paradox . . . . . . . . . . . . . . . . . 6

2.3 Braess' paradox in TASEP networks . . . . . . . . . . . . . . 7

2.4 Types of traffic information . . . . . . . . . . . . . . . . 10

2.4.1 Traffic Information Available in Present Day Road Networks . . . 11

2.5 Previous research on route choices . . . . . . . . . . . . . . . . . 12

2.5.1 Analysis of real world data . . . . . . . . . . . . 12

2.5.2 Mathematical models and simulation studies . . . . . . . . . . 13

2.5.3 Laboratory experiments . . . . . . . . . . . . . . . . . 14

2.5.4 How research from the different areas connects . . . . . . . . . 14

3 Route choice algorithms $\quad \mathbf{1 5}$

3.1 Public historical information . . . . . . . . . . . . . . . . 16

3.2 Public predictive information ................... 17

3.3 Personal historical information . . . . . . . . . . . . . . 17

4 Simulation results $\quad 18$

4.1 Public predictive information. . . . . . . . . . . . . . . 20

4.2 Personal historical information. . . . . . . . . . . . . . . 24

5 Conclusions $\quad 28$

\begin{tabular}{l|l|} 
Appkixddid Aser optima in Braess' original example & 29 \\
\hline
\end{tabular}

ApphrediesB states 30

Appecadixacy of the travel time predictions used for public predictive information $\quad[32$

\section{Introduction}

Everyday experience shows that we spend a lot of time in traffic jams [1 3] . This time can add up to more than $100 \mathrm{~h}$ per year in certain parts of the world [4]. Two potential solutions to the problem of congestions come to mind: 1) building more roads and 2) providing traffic information for the drivers to make better decisions. However, it is known for some time that new roads are not necessarily a solution. The Fundamental Law of Road Congestion [4] states that more roads will lead to more road users which will lead to congestions on the new roads as well. 
Even if the traffic volume does not increase, new roads will not necessarily lead to an improvement, e.g. shorter travel times for the drivers. This paradoxical fact has been established rigorously 50 years ago by Braess [5, 6] and is now commonly known as Braess' paradox. In compact form it can be formulated in the following way:

In road networks of selfish users additional roads can lead to higher travel times for all users.

Although it is formulated using terminology from traffic engineering, the paradox has been shown to occur in a variety of other systems as well, ranging from general transport networks [7,9] to mechanical and electrical systems [10, 11], pedestrian dynamics [12], microfluidic networks [13], oscillator networks and power grids [14, 15]. A review of some examples from mechanical systems, biological networks, to power grids can be found in [16].

Braess has exemplified the paradox for a simple network that has just five edges. One of the essential ingredients for the occurrence of the paradox is that one has to distinguish two different kinds of optima in the system, the user optimum (uo, also called Nash equilibrium), and the system optimum (so) [17]. The uo is realized if network users distribute themselves onto the routes such that the travel times of all used routes are equal and lower than those of any unused routes. It reflects the perspective of individual drivers who can not improve their travel times by choosing a different route and is thus widely considered to be the stable state of a traffic network used by selfish users. The so corresponds to a global perspective, e.g. of an engineer or politician. In the so the drivers are distributed onto the routes such that a global parameter is minimal. Several different global parameters could be considered. Prominent examples are the weighted average of all travel times [17] or the sum of the travel times of all drivers [18]. Braess considered yet another definition of the system optimum: in his work [5, 6] as well as in our previous works on the Braess paradox [19, 20] and also in the present article the system optimum is defined as the state which minimizes the maximum travel time of all used roads. Indeed, the uo and the so can be different in certain situations, i.e. correspond to a different distribution of drivers on the available routes.

Braess' example [5, 6] consists of simple networks with 4 and 5 links (where links represent roads), respectively. In the network with 4 links, the uo and so coincide. The addition of a new road (i.e. the fifth link) changes the network such that the uo and so become different, or - more specifically - in the network that includes the new road, the individual travel times in the uo are larger than those in the so. The travel time functions are chosen in such a way, that the travel times in the uo of the network with the fifth link are higher than those in the network without the fifth link.

Although the paradox considers a simplified scenario, it has been observed in several real world situations. Newly built roads can worsen the traffic situation, or inversely, the closing of roads can improve traffic [21 23]. A concept related to Braess' paradox is the price of anarchy. It measures the reduced efficiency of a system due to the selfish behavior of agents [24]. 
Braess' original work is based on a macroscopic mathematical model of freeway traffic. A lot of further research effort was made and let to a more coherent understanding of the paradox in such mathematical models [7 [9, 18, 25 27]. In previous works [19, 20] we have studied the original Braess network with a more realistic traffic dynamics that e.g. includes stochastic fluctuations. In these microscopic models, all cars are considered individually. By varying the route choice decisions of the drivers, the user optima of the networks with and without the new road were found. The travel times in the user optima of the two different versions of the networks were then compared. These systems show rich phase diagrams which include extended regions where Braess' paradox can be observed. More specifically, two different cases of the drivers' route choice decisions have been distinguished: 1) routes are chosen stochastically by each driver [19], and 2) drivers use fixed strategies for their route choices [20].

In the aforementioned research based on macroscopic and microscopic models, Braess' paradox is found in the sense that, for the same amount of cars, user optima exist such that those of the networks with the new road have higher travel times than those of the corresponding networks without the new road. For the case of perfectly rationally deciding drivers with access to perfect traffic information, one can assume that these user optima would indeed be realized and that thus the Braess paradox would really be observed. The question that remains is if those potentially accessible user optimum states are also realized in more realistic situations with real (imperfectly deciding) human network users who base their decisions on real (imperfect) traffic information.

In fact it has been shown that often travel time minimization is not the only factor determining route choice decisions [28 30] and that even if it is, drivers do not decide perfectly rational on this basis [31, 32]. Therefore variations of the above mentioned definition of the user optimum were introduced [33, 34]. While perfect traffic information is also not present in road networks, with the introduction of smartphone routing apps and personal navigational systems, more accurate information is available [35]. It has recently been shown, that this might actually lead to the realisation of user optima in some cases of road networks [36, 37].

Here we examine whether Braess' paradox is realized in a microscopic transport model if users decide intelligently based upon information similar to that available for real modern day road networks. This is meant in the following sense: are the user optima in the systems with and without the new road which are accessible by externally tuning the users' decisions reached if users intelligently base their decisions on information similar to that available in present day real world networks. Two types of traffic information are considered: information based on the drivers own memory or experience (as e.g. in a commuter scenario) and information similar to that provided by smartphone apps. It is shown that both types drive the system into its user optima, realizing Braess' paradox. This is a strong indication for answering the question if the paradox still occurs in present day road networks or if it is potentially resolved due to modern traffic information: it seems that the paradox is indeed still of great importance! It is furthermore shown that user optima of different phases of the system (that do not 
show Braess' paradox) are also realized. We conclude that the phase diagrams derived in [19, 20] are 'realistic'.

\section{Background information}

Before presenting the results of our study we define some terminology and provide some more details about Braess' paradox and some background to traffic information and route choice processes. Then we define the model investigated here and give a short summary of previous results.

\subsection{Some terminology}

Since this paper is concerned with traffic networks we will use the terms "edge" and "road" interchangeably. A "route" is a connection between an origin and a destination in a traffic network. A route can be comprised of multiple roads and also of so-called "junction sites" which connect roads. The "travel time" of a road refers to the time it takes to traverse the road. The travel time on a route refers to the time needed to traverse the route, i.e. to get from the origin to the destination on that route. Furthermore, the terms "car" and "particle" are used interchangeably as well as "driver", "user" and "agent".

A "strategy" of an agent refers to its route choice. Two specific types of strategies that were used in our previous research [19, 20] are "pure strategies" and "mixed strategies". For pure strategies the driver chooses exactly one specific route, whereas "mixed strategy" refers to the case in which one route is chosen out of several routes with a certain probability. In real road networks, if a network user has to perform route choices repeatedly, e.g. mixtures of these two strategy-types can be at play. A "state" of the network is given by the distribution of the cars onto the routes, i.e. the set of the strategies of all drivers.

The "user optimum" state is often considered to be the stable state of traffic networks with "selfish users", i.e. agents who choose their routes non-altruistically only according to their own intentions. The user optimum is reached if the travel times of all cars are such that they are equal on all used routes and, at the same time, lower than those of any unused routes [17]. A "pure user optimum" (puo) is reached if all agents follow pure strategies. In this case the numbers of cars using each route are integer numbers (or zero). This corresponds to a "pure Nash equilibrium" in game theory [38]. A "mixed user optimum" (muo) is reached if all agents follow mixed strategies and the mean values of the travel times of all used routes are equal and lower than those of any unused routes. In this case the average numbers of cars following routes can be positive non-integer values or zero. It corresponds to a "mixed Nash equilibrium" [38]. 


\subsection{The Braess paradox}

The original example. The network proposed by Braess in his original work [5, 6] is shown in Fig. 1. In his scenario all agents move from the same origin to the same destination. Road 5 is the road which is added to the system. Thus in the network without road 5, which we call "4link network" from now on, there are two routes from origin to destination: route 14 and route 23 . In the network with road 5, called "5link network" in the following, there is the additional route 153 to the destinationt. In the

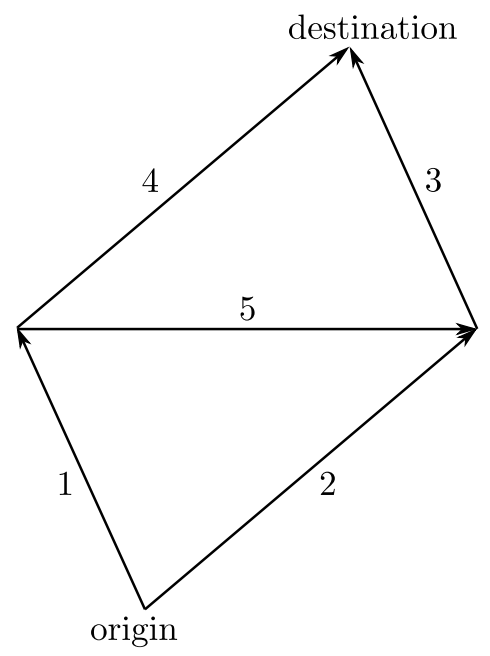

Figure 1. Braess' network as presented in his original work [5, [6]. All agents move from the same origin to the same destination. There are three routes available, named after the edges they are comprised of: routes 14, 23 and 153. Edge 5 is supposed to be newly added to the network. Route 153 is only available after this addition. The networks without and with edge 5 are called 4 link and 5link networks, respectively.

original example, traffic flow was characterized only by travel time functions $T_{i}$ linear in the number of cars $n$ using road $i$ :

$$
\begin{aligned}
& T_{1}=T_{3}=10 n, \\
& T_{2}=T_{4}=50+n, \\
& T_{5}=10+n .
\end{aligned}
$$

The 4link network is thus symmetric.

Braess showed that for a total number of $N=6$ cars, the pure user optimum of the 4link network is given by half the cars using route 14 and the other half route 23, respectively: $n_{14, \text { puo }}^{(4)}=n_{23, \text { puo }}^{(4)}=3$. This results in equal travel times of both routes: $T_{\text {puo }}^{(4)}=T_{14, \text { puo }}^{(4)}=T_{23, \text { puo }}^{(4)}=83$. In the 5link network the pure user optimum is given by $n_{14, \text { puo }}^{(5)}=n_{23 \text {,puo }}^{(5)}=n_{153 \text {, puo }}^{(5)}=2$ with equal travel times on all routes, $T_{\text {puo }}^{(5)}=T_{14, \text { puo }}^{(5)}=T_{23 \text {,puo }}^{(5)}=T_{153 \text {,puo }}^{(5)}=92$. Thus, $T_{\text {puo }}^{(5)}>T_{\text {puo }}^{(4)}$, i.e. the new road leads to larger pure user optimum travel times.

$\ddagger$ Routes are named according to the (ordered!) edges they are comprised of. From here on, variables corresponding to the 4link and 5link networks are marked with superscripts (4) and (5), respectively. 
The paradox is also observed if all cars follow mixed strategies $\S$. For $N=6$, in the 4link network the mixed user optimum state is found if routes 14 and 23 are chosen with equal probabilities: $p_{14, \text { muo }}^{(4)}=p_{23, \text { muo }}^{(4)}=1 / 2$. This leads to equal travel time expectation values of $\left\langle T_{\text {muo }}^{(4)}\right\rangle=\left\langle T_{14, \text { muo }}^{(4)}\right\rangle=\left\langle T_{23 \text {,muo }}^{(4)}\right\rangle=88.5$. In the 5link system the mixed user optimum is given for $p_{14, \text { muo }}^{(5)}=p_{23 \text {, muo }}^{(5)}=5 / 13$ and $p_{153 \text {,muo }}^{(5)}=3 / 13$ with travel time expectation values $\left\langle T_{\text {muo }}^{(5)}\right\rangle=\left\langle T_{14, \text { muo }}^{(5)}\right\rangle=\left\langle T_{23 \text {,muo }}^{(5)}\right\rangle=\left\langle T_{153 \text {,muo }}^{(5)}\right\rangle=93.6923$. Thus also in the case of mixed user optima, $\left.\left\langle T_{\text {muo }}^{(5)}\right\rangle\right\rangle\left\langle T_{\text {muo }}^{(4)}\right\rangle$.

Results of further research. Since the initial description of the paradox by Braess a lot of efforts were made to understand the phenomenon in more detail in the context of mathematical models of traffic networks. It was shown that in the original model of Braess, the paradox occurs for several amounts of total users, and not only for Braess' specific example of $N=6$ [9]. The paradox also occurs for different choices of (linear) travel time functions in Braess' original network [25] and also in different network topologies [18]. A general framework for predicting the occurrence of the paradox in networks of uncorrelated links was established [7, 26]. Mathematical models including correlations between the roads were studied in [8]. It was furthermore shown for arbitrary networks and models with monotonically increasing travel time functions, that if the paradox occurs at a certain density, the new road will be ignored completely for densities higher than a certain threshold [27].

\subsection{Braess' paradox in TASEP networks}

In an attempt to get an understanding of the paradox in a more realistic context, in two recent articles [19, 20] we have shown that the Braess paradox can also be observed in networks of stochastic, microscopic traffic models, i.e. in networks of totally asymmetric exclusion processes (TASEPs). The description of traffic flow in Braess' original example, as summarized above, was rather basic, being more of a proof of principle instead of a realistic model. Braess used only linear travel time functions which is not realistic. In addition, microscopic interactions and the stochastic nature of traffic were omitted. Furthermore, correlations between the roads were not taken into account.

Modelling traffic flow in the network by coupled TASEP segments is a first step to a more realistic traffic description by including these aspects while, at the same time, keeping the system simple enough to be analysed. There is a vast amount of research dedicated to the many variants of TASEPs and their properties. For some condensed information the reader is referred to e.g. [39 41].

The Braess network of TASEPs for two different route assignment types is shown in Figure 2. The network has the same structure as Braess' original network (Fig. 1). The edges $E_{i}$ are now made up of TASEPs of lengths $L_{i}$ joined through junction sites $j_{k}$. Furthermore we use periodic boundary conditions via the additional link $E_{0}$. This

$\S$ For a more detailed discussion of the mixed user optima, see Appendix A. 
(a)

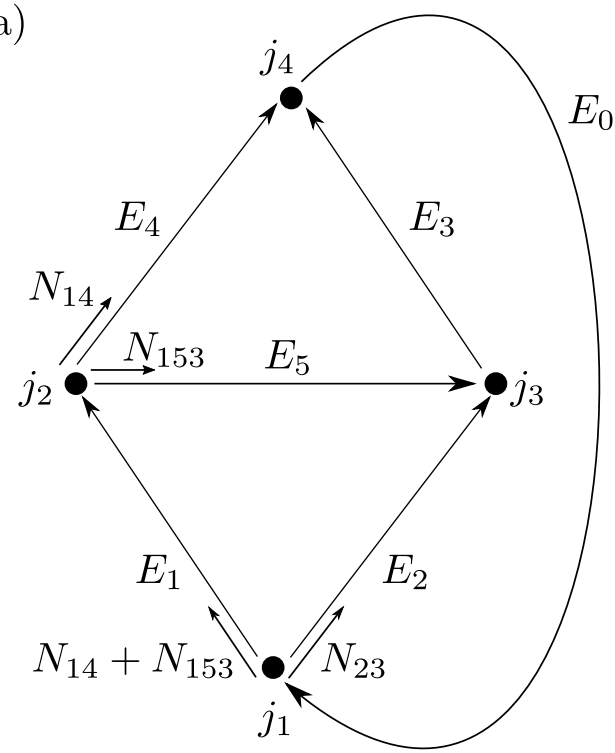

(b)

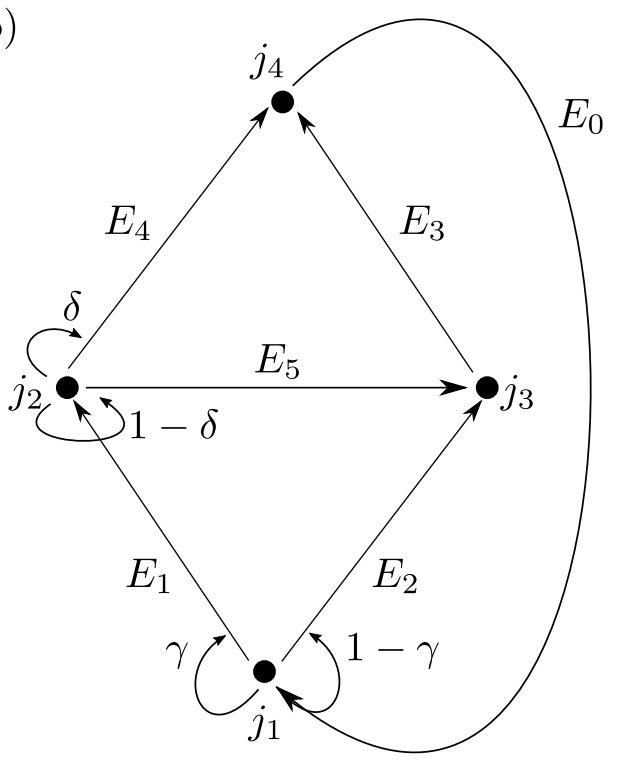

Figure 2. Braess' network of TASEPs with periodic boundary conditions for two different types of externally tuned strategies. The structure of the network corresponds to that used by Braess in his original article, as shown in Figure 1. Here, edges $E_{1}$ to $E_{5}$ are made up of TASEPs, coupled through junction sites $j_{1}$ to $j_{4}$. The added edge $E_{0}$ realizes the periodic boundary conditions. Part (a) shows the system with fixed strategies, in which fixed numbers of cars $N_{i}$ use routes $i$ as studied in 20]. In part (b) the system with turning probabilities, as studied in [19], is shown. Particles sitting on junctions $j_{1}$ or $j_{2}$ turn left with probabilities $\gamma$ and $\delta$, respectively.

has the advantage that the total number of particles $M$ is conserved which allows to compare the travel times of the 4link and 5link systems in their respective user optima for the same number of particles. To reduce the number of parameters, in the following we will present results only for the following edge lengths:

$$
L_{1}=L_{3}=100, \quad L_{2}=L_{4}=500, \quad L_{0}=1 .
$$

The length of $E_{5}$ will be varied, subject to the condition that the length of the new route is smaller or equal to that of the two old routes (which are of equal length).

Figure 2 (a) shows the network with fixed personal strategies as analysed in [20]. In this case, each particle keeps its personal pure strategy of always choosing one specific route. Numbers of $N_{14}, N_{23}$, and $N_{153}$ particles choose routes 14, 23 and 153 respectively, with $N_{14}+N_{23}+N_{153}=M$. The three numbers $N_{14}, N_{23}$, and $N_{153}$ can also be expressed through the two quantities

$$
\begin{aligned}
& n_{\mathrm{l}}^{\left(j_{1}\right)}=1-\frac{N_{23}}{M}, \\
& n_{\mathrm{l}}^{\left(j_{2}\right)}=\frac{N_{14}}{N_{14}+N_{153}},
\end{aligned}
$$

which describe the fraction of particles turning 'left' at junctions $j_{1}$ and $j_{2}$. User optima can be found by varying the $N_{14}, N_{23}, N_{153}$. The user optima obtained in this scenario are pure user optima. 
In Figure 2 (b), the network with the route assignment procedure governed by turning probabilities, as studied in [19], is shown. In this case all particles are equal. A particle on junction $j_{1}$ jumps to the left (i.e. onto $E_{1}$ ) with probability $\gamma$ and to the right (i.e. onto $E_{2}$ ) with probability $1-\gamma$. In the 5link network, particles on junction $j_{2}$ jump left (i.e. onto $E_{4}$ ) and right (i.e. onto $E_{5}$ ) with probabilities $\delta$ and $1-\delta$, respectively. User optima in this network are found by varying the $\gamma$ and $\delta$. They are mixed user optima.

These two types of route assignment are from here on called "externally tuned strategies". This is meant in the sense that all decisions are set at the beginning of each simulation run externally, i.e. not intelligently by the particles themselves. These route choices will later on be distinguished from route choice decisions made by 'intelligent' agents following our route choice algorithm.

The phase diagrams for both networks are shown in Fig. 3, The phase of the system depends on the ratio $\hat{L}_{153} / \hat{L}_{14}$ of the lengths of the new route $153, \hat{L}_{153}$, and the two old routes, $\hat{L}_{14}=\hat{L}_{23}$; and the global density. Since the phase of the system describes how the travel times of the 4link and 5link systems' user optima are related, the global densities of both systems $\rho_{\text {global }}^{(4)}=M /\left(4+\sum_{i=0}^{4} L_{i}\right)$ and $\rho_{\text {global }}^{(5)}=M /\left(4+\sum_{i=0}^{5} L_{i}\right)$ are shown.

(a)

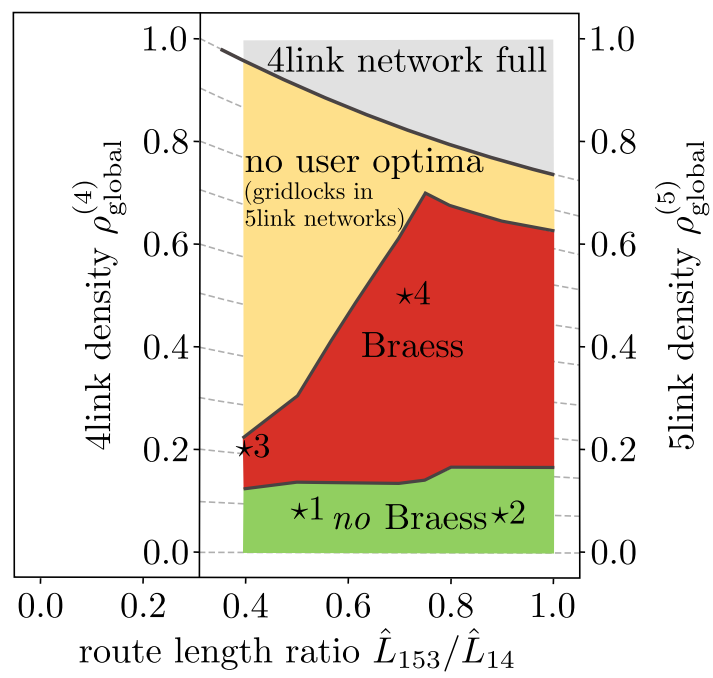

(b)

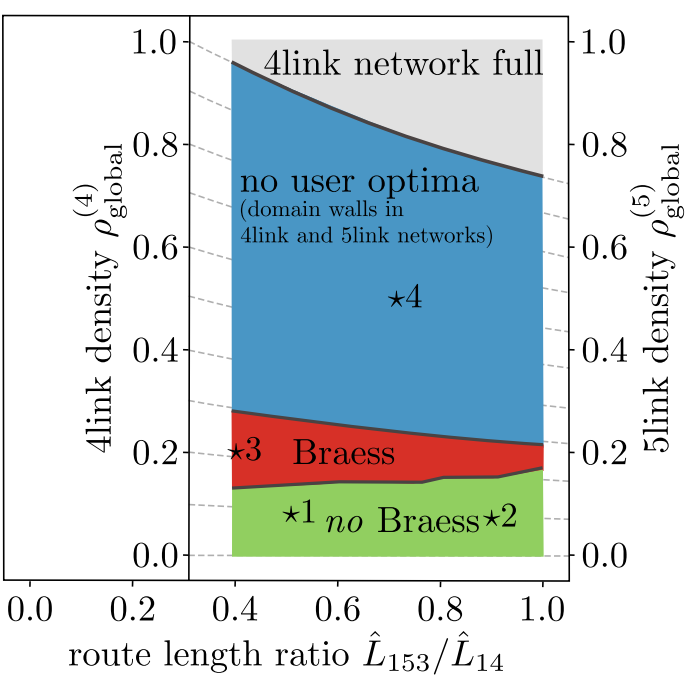

Figure 3. Phase diagrams of Braess' network of TASEPs (Fig. 2) for (a) fixed route choices and (b) turning probabilities. The phase of the system depends on the route length ratio $\hat{L}_{153} / \hat{L}_{14}$ between the new and old routes and the global densities $\rho_{\text {global }}^{(4 / 5)}$ in 4 link/5link systems. In both phase diagrams four states $\star 1$ to $\star 4$ are marked. The route choice algorithm presented in the present paper is tested on these states (Sec. (4)). The detailed parameters of the four states are described in Appendix B. The shown phase diagrams are simplified, as e.g. sub-phases are not shown. For full details, see [19, 20].

In both systems at low densities the user optima in the 5link networks have lower travel times than those of the corresponding 4link networks, thus the system is not in a 
Braess phase ("no Braess"/green phases). The Braess paradox is observed in significant parts of the phase spaces of both systems ("Braess"/red phases). In both systems at higher densities no user optima could be found due to gridlocks (orange phase) and fluctuating domain walls (blue phase) in the system with fixed strategies and turning probabilities, respectively. For more detailed phase diagrams and explanations, the reader is referred to [19, 20].

The phase diagrams were obtained by assigning the particles onto their routes externally via the $\left(N_{14}, N_{23}, N_{153}\right)$ and $(\gamma, \delta)$. By varying these parameters, user optima were found and the travel times of the 4link and corresponding 5link systems were compared to construct the phase diagrams. In the present article we address the question if these user optima are actually realized if the particles choose their routes intelligently, based on different kinds of information. As in the original example presented by Braess in his mathematical model, the pure and mixed user optima for one combination of the $\left(\hat{L}_{153} / \hat{L}_{14}, \rho_{\text {global }}^{(4 / 5)}\right)$ do not necessarily coincide, i.e. the $\left(n_{1}^{\left(j_{1}\right)}, n_{1}^{\left(j_{2}\right)}\right)$ and $(\gamma, \delta)$ which realize a pure and mixed user optimum respectively do not always have equal values.

In the following, we summarize some results of previous research on traffic information and route choice processes and then present our route choice algorithm. This algorithm is then applied to the four states marked in Figure 3. The travel times of the user optima of these states can be found in Appendix B.

\subsection{Types of traffic information}

Information about the state of traffic networks is called traffic information. It can consist of information on various aspects of the traffic network, such as the positions of all vehicles, average speeds, traffic light phases and many more. Here we focus specifically on information about travel times on roads and routes in the network.

Information available to network users can be grouped into two main categories.

(i) "Public Information" is in principle accessible to all network users.

(ii) "Personal Information" is only known to individual network users. It is usually based on the user's personal experience and/or specifically designed for a specific user, e.g. based on his/her current position, destination etc.

These two main categories can contain information from three different subcategories [42].

a) "Historical Information" describes travel times measured in the network in previous time periods.

b) "Current Information" refers to the most up-to-date information available. It can be given in the form of providing network users with the current state of the network, e.g. providing the current traffic densities or the currently measured (average) speeds on certain routes as e.g. in [43].

If one sticks strictly to this definition, in real traffic networks travel time information cannot be current information. This is due to the following problem: if e.g. a 
network user finishes a trip in a given moment and her experienced travel time information is immediately made available to the public, this information does not represent the travel time in the network right in that moment. It is instead the travel time of the used route at the current time minus the measured travel time. In the current moment the traffic situation might have changed and a user choosing the same route right now might experience a different travel time.

c) "Predictive Information" is e.g. given in the form of estimated travel times for the routes. In contrast to the two other types of information, predictive information can - by its nature - not be guaranteed to be accurate. If predictive information is given to network users in the context of route choices, a specific dilemma occurs: the information potentially influences the agents, leading them to take certain route choice decisions which then change the traffic state and thus invalidate the information [44]. A special kind of predictive information is "prescriptive information" which - opposed to "descriptive information" which only describes network states - advises network users to use specific routes [35].

Network users may have access to combinations of all these types of information and make their own individual route choice decisions based upon them.

\subsubsection{Traffic Information Available in Present Day Road Networks In present day} road networks various kinds of information are available. Individual network users might have personal historical information about travel times on specific routes based on their own experiences if they used these routes before (personal historical information, in this case also called "experiential information" [35]). This is often the case in commuter scenarios. Furthermore, individual users might have some insights from friends or other personal sources. Public information of various kinds is available from numerous sources. Public historical information can be found in internet databases and is also available in various smartphone routing apps and personal navigational systems. Current public information is available from radio traffic forecasting and various advanced traffic information systems (ATIS) [45] such as variable road signs.

Personal navigational systems and smartphone routing apps also provide predictive public information. This type of information is considered public since these devices are in principle accessible for everyone. In contrast to the former mentioned information, these tools also provide prescriptive route choice information. Among many alternatives, Google Maps was the most popular routing app in the US in 2018 [46]. The main difference from more traditional types of traffic information is that such apps rely on crowdsourcing [47]. This means that all users of the app send their location data to Google where this data is in turn used to get an accurate picture of the current traffic situation of the network (given there are enough Google Maps users in the network at that moment). This current information is combined with large quantities of historical information to provide fairly accurate public predictive (prescriptive) information (about travel times). Details about how the Google Maps algorithm works are not known to 
the public [47, 48].

To approximate the situation which is found in real modern road networks in this article we consider user decisions based on personal historical and public predictive information. A mixture of these two types of information is found in real road networks, especially in commuter scenarios.

The detailed knowledge of current traffic states available due to crowd sourced information could also be used to provide information to road users that aims at optimizing the state of the whole traffic system. Such information would be given in different forms than just information about (predictive) travel times on available routes. It could be designed e.g. to drive traffic networks into their system optima and could thus lead to a reduction of traffic congestion, as shown e.g. in [49]. Even though the necessary data for such information is in principle available, such systems are (to our knowledge) currently not in use, which is why they are not considered here.

\subsection{Previous research on route choices}

The question how road users choose their routes given certain types of traffic information and consequently the question if user optima are realized in networks of selfish users has been analysed in various scientific disciplines. The research approaches can be subdivided into three groups: analyses of real world data, mathematical models and simulation studies, and laboratory experiments.

2.5.1. Analysis of real world data Since traffic networks are generally highly complex structures with numerous users that all decide individually it is difficult to gain objective knowledge about what drives the decision making processes in these networks. Next to much anecdotal knowledge about route choices, some large scale real world observations and experiments were performed. A study from 2001 [29] hints at travel time minimization not being the only factor driving route choice decisions. Furthermore, travel time seems to be systematically misperceived by many drivers [28]. In a study in which vehicles of a large number of network users were equipped with GPS units it was shown that only approximately one third of traffic network users chooses the fastest available path [30]. For a nice recap of results of previous research, the reader is also referred to [30].

The introduction of smartphone routing apps lead to a different usage of road networks in many parts of the world. Data suggests that minimal travel time becomes more important when using those apps and hints at the realization of user optima due to the heavy usage of these tools [37]. There are also many negative side-effects of such tools, such as increasing use of smaller side roads to avoid congested main roads, leading to complaints by residents [50].

An effect that has already been predicted in mathematical traffic models [42, 51]

and queueing models [52] and was observed in simulations [44] has also been observed as a consequence of routing apps: routing apps provide predictive information about 
travel times based on the current network state to all users. This information may then influence the users' decisions in such a way that these decisions invalidate the prediction. This so-called "overreaction" [42] has been observed in networks with routing apps [37].

2.5.2. Mathematical models and simulation studies In earlier days, research on route choices was performed using mathematical models [42, 51, 53] which already predicted many effects that were later confirmed e.g. by simulations. In [54, 55] the Braess paradox was considered in a discrete time macroscopic mathematical model. It was shown that in the given model, the user optima leading to Braess' paradox can be avoided with a special kind of personal historical information.

For route choice research on the basis of microscopic simulations it has proven to be useful to implement so-called "multi-agent techniques" [44, 56]. The traffic flow itself, forming the "tactical layer", is modelled by a stochastic microscopic traffic model. The acquisition of information and the route choice decisions, the so-called "strategic layer", are modelled by an algorithm. This multi-agent approach is also used in the present paper.

Multi-agent models with the tactical layer being described by the NagelSchreckenberg model [57] have been studied for certain types of traffic information: It was shown that the availability of public historical information in a symmetric two-route network with open boundary conditions leads to oscillations around the user optimum [44]. In [44] the latest experienced travel times on both routes were made available to all network users. This leads to overreactions since this travel time information is based on the network states previous to when the information is made available. The user optimum is reached when agents choose each route with equal probability. Instead oscillations between periods of all cars using just one route and times of all cars using just the other route are realized. This observation lead to the proposition of many other types of information with the aim of realizing the user optima in this specific network (see e.g. 58 60]). A good review of these information types and how they perform is found in [61]. There it is also pointed out that most of these realize user optima only in the specific symmetric two route scenario. It was also shown that the paradox can be observed in the Braess network, if traffic flow is modelled by the Nagel-Schreckenberg model [55].

In [62] a two route model with periodic boundary conditions and dynamics similar to TASEP is studied with users with personal historical information, i.e. users that have memories of certain lengths that decide based on their own experiences. It is shown that this type of information realizes user optima in the network. This is similar to some of the results to be presented later in the present paper.

Large-scale simulations of systems with information similar to that provided by smartphones suggest that user optima are realized in these systems [37]. To our knowledge no models based on simulations in connection with personal historical information or public predictive information like that provided by smartphones, implemented in the way given in the present paper, have been studied in controlled 
small networks like the present Braess network.

2.5.3. Laboratory experiments Next to research based on mathematical models and simulations which was mainly conducted in the traffic science and traffic engineering community, in the social sciences and behavioral economics many laboratory experiments on route choice processes were performed with the aim of understanding how humans decide. Typically, a road network is implemented and the traffic flow is described by a mathematical model. Human subjects are then asked to perform route choices repeatedly given various types of information. Usually real money is paid out as an incentive to perform well in the task of travel time minimization.

A nice review of route choice experiments is found in [63]. Here we focus on experiments that either directly address the Braess paradox or are closely related. In 32] and [35] scenarios with two and three unconnected links from origin to destination were studied, respectively. In both studies, when participants relied on personal historical information, i.e. their own experience of travel times of previous rounds, user optima were reached on average with some persisting fluctuations. In [32] also the situation with public historical information, in which participants had knowledge of travel times also on routes not taken, was studied. The user optimum was also reached on average.

In [64] route choice decisions in the 5link version of the Braess network were studied in a virtual experiment. Participants had to chose routes daily in the app "WeChat". Subject to public historical information the user optimum was reached.

In [31] the Braess paradox was tested directly in the laboratory. Participants performed route choices first in Braess' 4link and then in the 5link network. Subject to public historical information user optima were reached in both cases (in the 4link on average, while fluctuations decreased in the 5link) and the paradox was realized. In a further network of different topology, Braess' paradox was realized as well [31]. Furthermore, in [65] another network exhibiting Braess-behaviour was studied. The paradox was also realized here.

2.5.4. How research from the different areas connects The research performed in the different scientific disciplines employing the various approaches mentioned above all add to the understanding of route choice processes while all of them have their advantages and disadvantages. In observations of real world data, typically the system cannot be controlled as well as in the toy systems studied in simulations and laboratory experiments. Here, there is always a larger underlying network. The objective of the 'participants' is not clear either. Nevertheless, on one hand, this lead to important discoveries such as the minimization of travel time not necessarily being the sole goal of network users. On the other hand, these conclusions cannot be proven rigorously, since the objectives and motivations for the route choice of the agents are not known.

The two more controlled approaches also differ in important ways: in all the works cited in the paragraph on laboratory experiments, the traffic description is limited to deterministic, macroscopic travel time functions of the individual roads in the network. 
Microscopic interactions are omitted and furthermore correlations between the roads are not modelled. Since these omitted details lead to a much more complicated traffic behavior in real traffic networks, as travel times could e.g. change more drastically if many drivers change their strategies, one can argue that drivers reactions could also be different in these cases. Thus it is not really clear if the laboratory observations really transfer to the real world. In research based on simulations, the tactical layer, i.e. the description of traffic flow, is more realistic, whereas the route choice decisions, i.e. the strategic layer, is not as realistic since it is not done by real humans.

Drawing from the observations of real world data one also has to note that the results from laboratory experiments cannot be taken as facts since in real world data it was observed that travel time minimization is not necessarily the main objective of drivers. In laboratory experiments nevertheless it is the sole objective by design of the experiment.

\section{Route choice algorithms}

To find out whether user optima are realized in the 4link and 5link versions of a Braess network of TASEPs (Fig. 2), when used by intelligently deciding agents, we implemented the following route choice algorithm. We examined variants where the agents have access to personal historical and public predictive information. With this we want to combine a more realistic tactical layer (i.e. a microscopic traffic modelled by coupled TASEPs) with a more realistic strategic layer with decisions based on realistic traffic information.

Due to the periodic boundary conditions all $M$ particles stay in the system and thus decisions based on personal experiences, i.e. on the memory of the particles, can be implemented.

The system is always initialized by placing $M$ particles on the routes randomly and assigning an initial pure strategy (to choose either route 14, 23 or 153) randomly to each agent. The system then undergoes a relaxation procedure which is different depending on the type of information used. The relaxation procedures are explained in the following paragraphs which describe the different types of implemented information. Once the system is relaxed, all agents have information about the (expected) travel times of all routes. The information for all three routes 14, 23 and 153 is from now on called $T_{14 \text {,info }}, T_{23 \text {,info }}$ and $T_{153 \text {,info }}$, respectively. If an agent went once from $j_{1}$ to $E_{0}$ (i.e. after jumping out of $j_{4}$ ), this agent has finished one "round".

After relaxation is complete, route choice decisions can occur at three points: before starting a new round (when 'sitting' on $E_{0}$ ) and during a round when sitting on $j_{1}$ or $j_{2}$ and not being able to jump to the desired target site. Before a new round, when sitting on $E_{0}$ before jumping to $j_{1}$, each particle generally chooses the route $i$ with the lowest $T_{i \text { info }}$. To make such decisions more realistic, the two following parameters are introduced to the algorithm.

(i) With probability $p_{\text {info }}$ the particle bases its decision on the available $T_{i \text {,info }}$. With probability $1-p_{\text {info }}$ the particle chooses one of the two or three routes (depending 
on whether the 4link or 5link system is simulated) randomly. The random decisions are introduced to account for the findings from observations in the real world, that users do not wholly base their decisions on the objective of minimizing travel times.

(ii) If, with probability $p_{\text {info }}$ as described in (i), an information-based decision will be taken, the difference between the expected travel times on the routes $\Delta T=$ $\left|T_{14 \text {,info }}-T_{23 \text {,info }}\right|+\left|T_{14 \text {,info }}-T_{153 \text {,info }}\right|+\left|T_{23 \text {,info }}-T_{153 \text {,info }}\right|$ is calculated $\|$. If this difference is below the threshold of $\Delta T_{\text {thres }}$, the agent stays on the route of the previous round. If $\Delta T \geq \Delta T_{\text {thres }}$, the agent switches to the route with the lowest $T_{\text {info. }}$ Thus the agents act "boundedly rational" 34 .

Additionally to these decisions before any new round, agents can make route choice decisions during the rounds. These decisions work as follows. Consider an agent in the 4link network sitting on $j_{1}$ who chose to take route 23 before the round began. If this agent cannot jump to its target site (first site of $E_{2}$ ) since this site is occupied, (s)he may re-decide for another route. If $T_{23 \text {,info }} \geq T_{14 \text {,info }}$ (agent chose route 23 based on a random decision before the round), (s)he will then immediately switch to route 14 . If $T_{23 \text {,info }}<T_{14 \text {,info }}$, the particle will keep trying to jump onto $E_{2}$ for $\kappa_{j_{1} \text {, thres }}$ times the to-be-expected saved time on route 23 , i.e. for $\kappa_{j_{1} \text {,thres }} \cdot\left(T_{14 \text {,info }}-T_{23 \text {,info }}\right)$ time steps. If after this waiting time a jump to its target site is not possible (s)he will switch to the other route.

This algorithm is slightly more complicated in the 5link system but works in the same sense: if an agent, due to a random decision, chose a route which does not have the lowest expected travel time and this route is blocked, (s)he will immediately decide for another route. If the chosen route does have the lowest expected travel time and the routes' entrance is blocked, on $j_{1}$ the agent will wait $\kappa_{j_{1} \text {,thres }}$ times the to-be-expected saved travel time before switching. In the 5link network, an analogous algorithm operates at junction $j_{2}$ where the parameter $\kappa_{j_{2} \text {,thres }}$ is introduced. For more details, see [66] where the algorithms for decision making are shown in pseudo code.

In the following the different types of information that are used for the algorithm are explained. Furthermore the relaxation procedures used in the simulations are explained.

\subsection{Public historical information}

Public historical information is implemented as follows: each time any user finishes one round (jumps out of $j_{4}$ ), the experienced travel time is recorded. This travel time is then made available to all agents as their $T_{\text {info }}$. This information is historical since, as explained in Sec. 2.4, the traffic state might have changed during the round. For this type of information a short relaxation process is needed: at the beginning all agents follow randomly assigned routes. As soon as each route has been used at least once, the system is considered to be relaxed. It has already been shown that this type of information does not lead to user optima in various two route scenarios but rather to very strong

\|I In the 4link system this expression reduces to $\Delta T=\left|T_{14, \text { info }}-T_{23, \text { info }}\right|$ 
oscillations around the user optimum (e.g. [44]). In [66] we show that the expected oscillations in the 4link system / two route scenario can be reproduced. Furthermore it is found that a similar behaviour occurs in the 5link / three route scenario. We do not present these results here, since they do not offer new insights. The interested reader is referred to [66].

\subsection{Public predictive information}

Public predictive information is provided on the basis of the current positions of all agents in the network. It is implemented as an approximation of the traffic information provided by smartphone apps in real road networks. To provide estimates of travel times for all edges, the densities $\rho_{i}$ are determined from the current number of particles on each edge $E_{i}: \rho_{i}=n_{i} / L_{i}$ where $n_{i}$ is the number of particles on edge $E_{i}$. From this density a travel time prediction $T_{i, \text { pred }}$ is calculated employing the formula

$$
T_{i, \text { pred }}=\frac{L_{i}}{1-\rho_{i}} .
$$

This equation is the exact stationary state expression for the travel time of a particle in a TASEP of length $L_{i}$ with periodic boundary conditions and density $\rho_{i}$ [19]. In our case it is only an approximation for the travel time on an edge: the edges neither have periodic boundary conditions nor are they (necessarily) in stationary states. It will show to produce reasonably accurate approximations (at least at low global densities) in the Braess network. From the approximated travel times of all edges the expected route travel times, which are used as the traffic information for all agents in the route choice algorithm, are calculated as

$$
\begin{aligned}
& T_{14, \text { info }}=T_{1, \text { pred }}+T_{4, \text { pred }}, \\
& T_{23, \text { info }}=T_{2, \text { pred }}+T_{3, \text { pred }}, \\
& T_{153, \text { info }}=T_{1, \text { pred }}+T_{5, \text { pred }}+T_{3, \text { pred }} .
\end{aligned}
$$

Here further approximations are introduced: potential waiting times at the junction sites are not considered and the system is only described in a mean field fashion since correlations are neglected.

At each decision point the current $T_{i \text {,info }}$ is calculated based on the current positions of all particles. This means that if an agent bases its decision on the $T_{i \text {,info }}$ before starting a round and then re-decides on one of the junctions, the $T_{i, \text { info }}$ might already have changed at the time of the second decision.

For this type of information no relaxation process is required, the agents are just placed on random positions in the network.

\subsection{Personal historical information}

Personal historical information is information based on the agents' experiences from previous rounds. Each agent is assigned a memory capacity of $c_{\text {mem }}$ rounds. For the last $c_{\text {mem }}$ rounds, each agent remembers which routes it took and their corresponding 
travel times. From these times the $T_{i \text {,info }}$ are calculated: they are the mean values of the travel times of each route as experienced in the last $c_{\text {mem }}$ rounds. Additionally, each agent remembers its last experienced travel times on all three routes. Like this, even if e.g. route 23 was not used in the last $c_{\text {mem }}$ rounds, the agent will still remember the travel time of that route from the last usage (that lies more than $c_{\text {mem }}$ rounds in the past).

For this kind of information there is a two-fold relaxation process. First, each agent is placed on a random position with a random strategy. Then it tries to gather one travel time value for each route. Once these values are obtained, the system keeps evolving until each agent has experienced $c_{\text {mem }}$ rounds. Once each agent used each route at least once and has a filled its memory of capacity of $c_{\text {mem }}$ rounds, the system is considered relaxed.

\section{Simulation results}

We applied the algorithms described in the previous section to determine the effects of the route choice behavior in the Braess network with TASEP-based traffic dynamics. The edge lengths $L_{0}, \ldots, L_{4}$ are given by Eq. (44). We focussed on four different combinations of $L_{5}$, the length of the added edge, and $M$, the number of agents. These are indicated by $\star 1$ to $\star 4$ in Figs. 3 (a) and (b). We applied the route choice algorithm to these states to see if user optimum states attainable by externally tuning all agents' strategies are realized. $\star 1$ and $\star 2$ have been chosen as representatives of states in which the new road is expected to improve the traffic situation: For both types of externally tuned strategies, i.e. for fixed numbers of agents following fixed routes and for all cars deciding according to the same turning probabilities, in states $\star 1$ and $\star 2$ the new road leads to a 5link system with lower user optimum travel times than in the corresponding 4 link system. State $\star 1$ is a special state since in the 5 link user optimum all cars use new route while the old routes have higher travel times and are not used. In the user optima of state $\star 2$ all routes are used. State $\star 3$ is a Braess state for externally tuned strategies: the user optima in the 5link networks have higher travel times than those of the corresponding 4link networks' user optima. For fixed personal strategies, in state $\star 3$ two user optima exist. State $\star 4$ is a Braess state for externally tuned strategies and fixed personal strategies. For externally tuned turning probabilities no (short term) user optima exist in this state since fluctuating domain walls are found in the system.

More details about the described states of the four points $\star 1$ to $\star 4$ are found in [19, 20]. Details for the exact parameters of the four states, their user optima and the corresponding travel times of these user optima can be found in Appendix B. These details are not essential if one is interested in qualitative effects, but might be helpful for a quantitative understanding of the following results.

For the obtained results which are discussed in the remainder of the present section, the parameters of the algorithm as introduced in Section 3 were always chosen to be:

$$
p_{\text {info }}=0.9
$$




$$
\begin{array}{ll}
\Delta T_{\text {thres }} & =10, \\
\kappa_{j_{1}, \text { thres }}=\kappa_{j_{2}, \text { thres }} & =0.1, \\
c_{\text {mem }} & =30,
\end{array}
$$

where $c_{\text {mem }}$ is only needed for the case of personal historic information. As we consider the continuous route choices in the Braess network to mirror a commuter scenario, it is reasonable to assume that in the majority of times, drivers choose their routes with the aim of minimizing their travel times, i.e. $p_{\text {info }}=0.9$. Furthermore it appears to be realistic that a commuter remembers approximately one month of her last experienced travel times $\left(c_{\text {mem. }}=30\right)$. Assuming that drivers may not switch from their preferred route if the expected saved time is really low, a value of $\Delta T_{\text {thres }}=10$ seems reasonable.

Figs. 411 show the results for all four states $\star 1$ to $\star 4$, each with public predictive and personal historical information. All figures have parts (a) and (b). Parts (a) show how the mean values of the travel times $\bar{T}_{i}$ of the routes $i$ develop with the system time where all times are measured in numbers of performed Monte Carlo sweeps. For details on the simulation process the reader is referred to [66]. The values of both routes in the 4link system and of the three routes in the corresponding 5link systems are shown for comparison. One can thus see if the new road leads to higher or lower travel times. Additionally the travel times that are expected from the pure and mixed user optima of the 4link and 5link systems with externally tuned strategies are shown for comparison by the dotted grey lines whose values are given by the $\tau_{i}$ on the second $y$-axis on the right.

Parts (b) of Figs. 411 show the two variables $m_{1}^{\left(j_{1}\right)}=1-M_{23} / M$ and $m_{1}^{\left(j_{2}\right)}=$ $M_{14} /\left(M_{14}+M_{153}\right)$ against the system time. Here, the $M_{14}, M_{23}$ and $M_{153}$ are the numbers of cars which follow routes 14, 23 and 153 at that system time. The values of the two variables $m_{1}^{\left(j_{1}\right)}$ and $m_{1}^{\left(j_{2}\right)}$ capture the fractions of agents using the three different routes. Similar to the variables $n_{1}^{\left(j_{1}\right)}$ and $n_{1}^{\left(j_{2}\right)}$, which we introduced in Eqs. (5), (6) in the context of externally tuned fixed personal strategies, they describe the fraction of particles turning 'left' of junctions $j_{1}$ and $j_{2}$, but in the present moment. They represent the strategies that the particles choose as a result of the route choice algorithm. Due to the algorithm their values can change before and during the rounds.

The $m_{1}^{\left(j_{1}\right)}$ and $m_{1}^{\left(j_{2}\right)}$ can be compared to the $\left(n_{1}^{\left(j_{1}\right)}, n_{1}^{\left(j_{2}\right)}\right)$ that realize the pure user optima for externally tuned fixed personal strategies and the $(\gamma, \delta)$ that realize the mixed user optima for externally tuned turning probabilities. Those strategies realizing the user optima by externally tuning the route choices are also shown by the dotted grey lines whose values are given by the $\sigma_{i}$ on the second $y$-axis on the right of parts (b) of Figs. 411. This allows to determine whether the algorithm drives the system into a user optimum. This gives an indication on how close it is to an expected pure or mixed optimum. To see if real pure user optima or real mixed user optima are realized, further analysis is needed: in a real pure user optimum no individual users would switch

I In the 4link system only $m_{1}^{\left(j_{1}\right)}$ is needed which reduces to $m_{1}^{\left(j_{1}\right)}=M_{14} / M$

+ In [66] these two variables are called implicit turning probabilities $\gamma_{\mathrm{imp}}$. and $\delta_{\mathrm{imp}}$. 
routes any more (this can not be seen from the $m_{1}^{\left(j_{1}\right)}$ and $m_{1}^{\left(j_{2}\right)}$, since they only show the sums of particles following specific routes). Situations without any individual particles switching routes can not be obtained in our algorithm since $p_{\text {info }}<1$. Still, if the number of switches is low one can presume that the algorithm brings the system close to a pure user optimum. In a mixed user optimum one would expect a higher number of individual route switches. To test if indeed a real mixed user optimum is realized a further statistical analysis of the behaviour of all individual particles is needed. Such an analysis is not shown here. The interested reader is referred to [66], where it is performed (in parts) for the state $\star 3$.

The results presented in the following all show single Monte Carlo simulations of the systems. They were confirmed in numerous runs of the same instances of the system with different RNG seeds, which showed the same behaviour apart from minor variations due to the stochasticity of the process.

\subsection{Public predictive information.}

The results for the algorithm with public predictive information are shown in Figs. 44 7 for the four different states $\star 1$ to $\star 4$. Fig. 4 shows results for state $\star 1$. One can see that the user optima of both the 4link and the 5link networks are realized in a stable manner. In the 4link network approximately half the agents choose route 14 and the other half route 23 (Fig. (4)(b)). Their mean travel times (Fig. 目(a)) equalize at the value expected from the user optima obtained in networks with externally tuned strategies. In the 5link network, apart from small fluctuations, almost all agents choose route 153 (Fig. 4 (b)) and this route has a lower travel time than the other two (almost unused) routes in the 5 link and also lower than those in the 4link system (Fig. 4 (a)). The " $E_{5}$ optimal, all 153 " state that is expected is thus realized.

The results for state $\star 2$ are shown in Fig. 5. In the 4 link system a user optimum is realized. We can see that approximately $M / 2$ agents use routes 14 and 23 without large fluctuations (Fig. [5 (b)). The mean travel times of the two routes in the 4link system thus equalize (Fig. 5 (a)). In the 5link system a different behaviour is observed. In Fig. (5) (b) it can be seen that the expected user optimum is reached on average. The

numbers of cars on the three routes (as represented through the $m_{1}^{\left(j_{1}\right)(5)}$ and $m_{1}^{\left(j_{2}\right)(5)}$ in Fig. [5 (b)) oscillate around the values expected from the $\left(n_{\mathrm{l}, \mathrm{puo}}^{\left(j_{1}\right)(5)}, n_{\mathrm{l}, \mathrm{puo}}^{\left(j_{2}\right)(5)}\right)$ and $\left(\gamma_{\text {muo }}^{(5)}, \delta_{\text {muo }}^{(5)}\right)$ from the pure and mixed user optima for externally tuned strategies. Due to these fluctuations the mean travel times of the three routes are close to each other but not equal (Fig. 5 (a)). Opposed to the expectation, the mean travel times of routes 14 and 23 are actually higher than those in the 4link system. Thus, even if the user optimum of the 5link system is realized on average, the algorithm with public predictive information drives the system into a state which is more of 'Braess nature' in the sense that the 5link travel times are higher than the 4link's, opposed to the expected " $E_{5}$ optimal" state.

Fig. 6) shows results for the case of public predictive information in state $\star 3$. As in the two previous states, the user optimum of the 4link system is realized. As seen in 

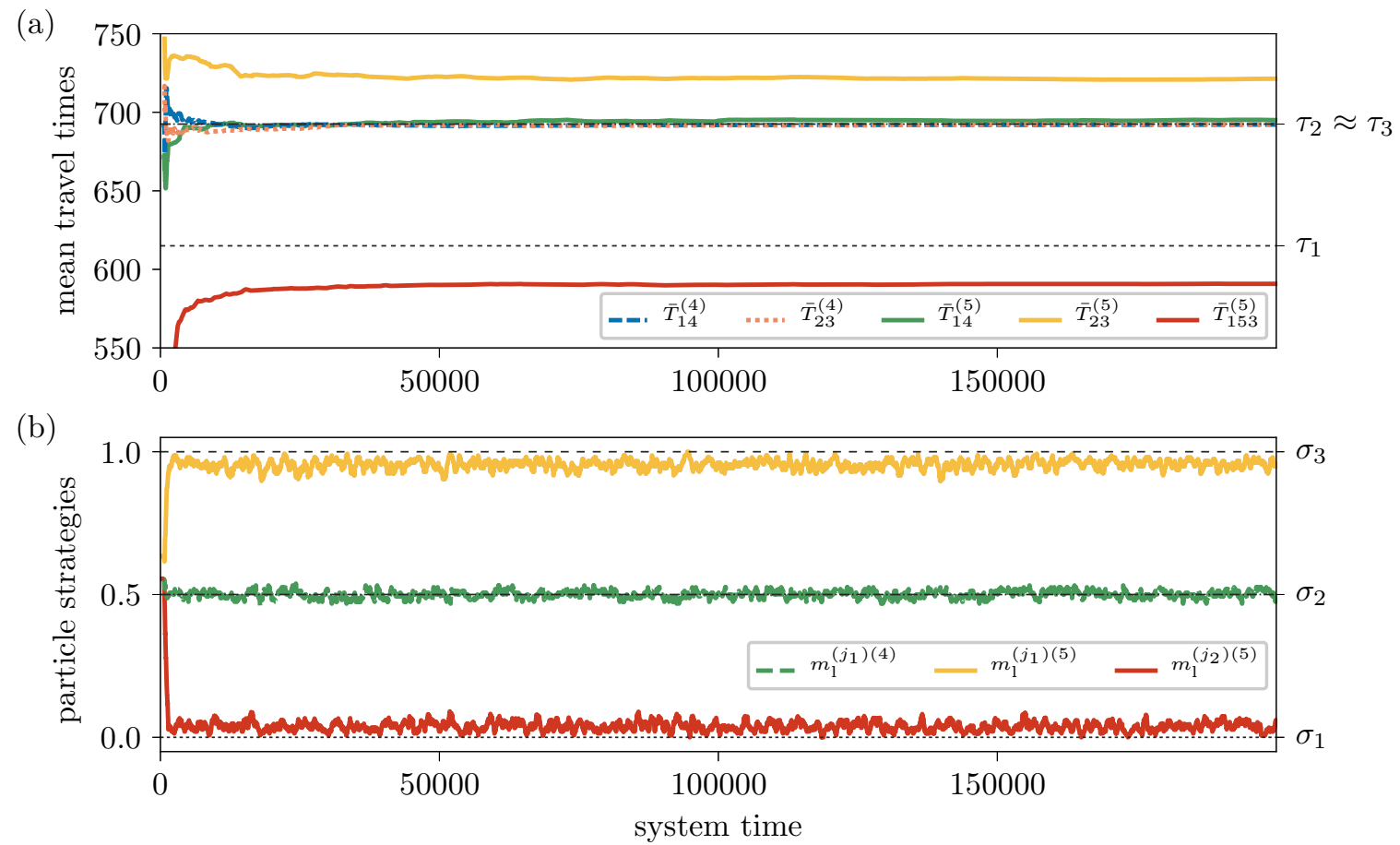

Figure 4. Results in state $\star 1$ if public predictive information is provided. As seen in (a), the mean travel times of both routes in the 4link system coincide with those from the expectations. The travel time of route 153 in the 5link is the lowest of the three routes. The expected travel times in the pure and mixed user optima found by externally tuning the strategies are shown by the dotted lines with $T_{\max , \text { puo }}^{(4)}=\tau_{2}, T_{\max , \text { muo }}^{(4)}=\tau_{3}, T_{\max , \text { puo }}^{(5)}=T_{\max , \text { muo }}^{(5)}=\tau_{1}$. Part (b) shows that in the 4link both routes are, as expected, used by equal amounts of agents, and in the 5link almost all cars use route 153 . The strategies realizing the user optima by externally tuning the strategies are given for comparison with $n_{1, \text { puo }}^{\left(j_{1}\right)(4)}=\gamma_{\text {muo }}^{(4)}=\sigma_{2}$, $\left(n_{1, \text { puo }}^{\left(j_{1}\right)(5)}, n_{1, \text { puo }}^{\left(j_{2}\right)(5)}\right)=\left(\gamma_{\text {muo }}^{(5)}, \delta_{\text {muo }}^{(5)}\right)=\left(\sigma_{3}, \sigma_{1}\right)$. The " $E_{5}$ optimal, all 153 " state is realized.

Figure 6 (b) half of the particles use routes 14 and 23. As can be seen in Figure 6 (a) the travel times of both routes equalize at the expected value. As detailed in Appendix B, the 5link system of state $\star 3$ with externally tuned parameters has two pure user optima and one mixed user optimum. The values of the $\left(n_{1, \text { puo }(\mathrm{i} / \mathrm{ii})}^{\left(j_{1}\right)(\mathrm{s})}, n_{1, \mathrm{puo}(\mathrm{i} / \mathrm{ii})}^{\left(j_{2}\right)(\mathrm{s})}\right)$ of neither of the two pure optima puo(i) and puo(ii) coincides with the values of the $\left(\gamma_{\text {muo }}^{(5)}, \delta_{\text {muo }}^{(5)}\right)$ of the mixed user optimum muo. In the 5link system the route choice algorithm produces strong fluctuations around the pure user optimum puo(ii) (Fig. 6 (b)), a state in which route 23 is not used and half the agents choose route 14 and the other half route 153 . Due to the fluctuations around the user optimum the travel times of the two used routes (route 14 and 153) are close to each other but not exactly equal (Fig.6(a)). They are all higher than those of the two routes in the 4link system. Thus a Braess state is realized. The almost unused route 23 has an even higher travel time, as expected.

Fig. 7 shows results for state $\star 4$. In the 4 link system the user optimum is realized. 

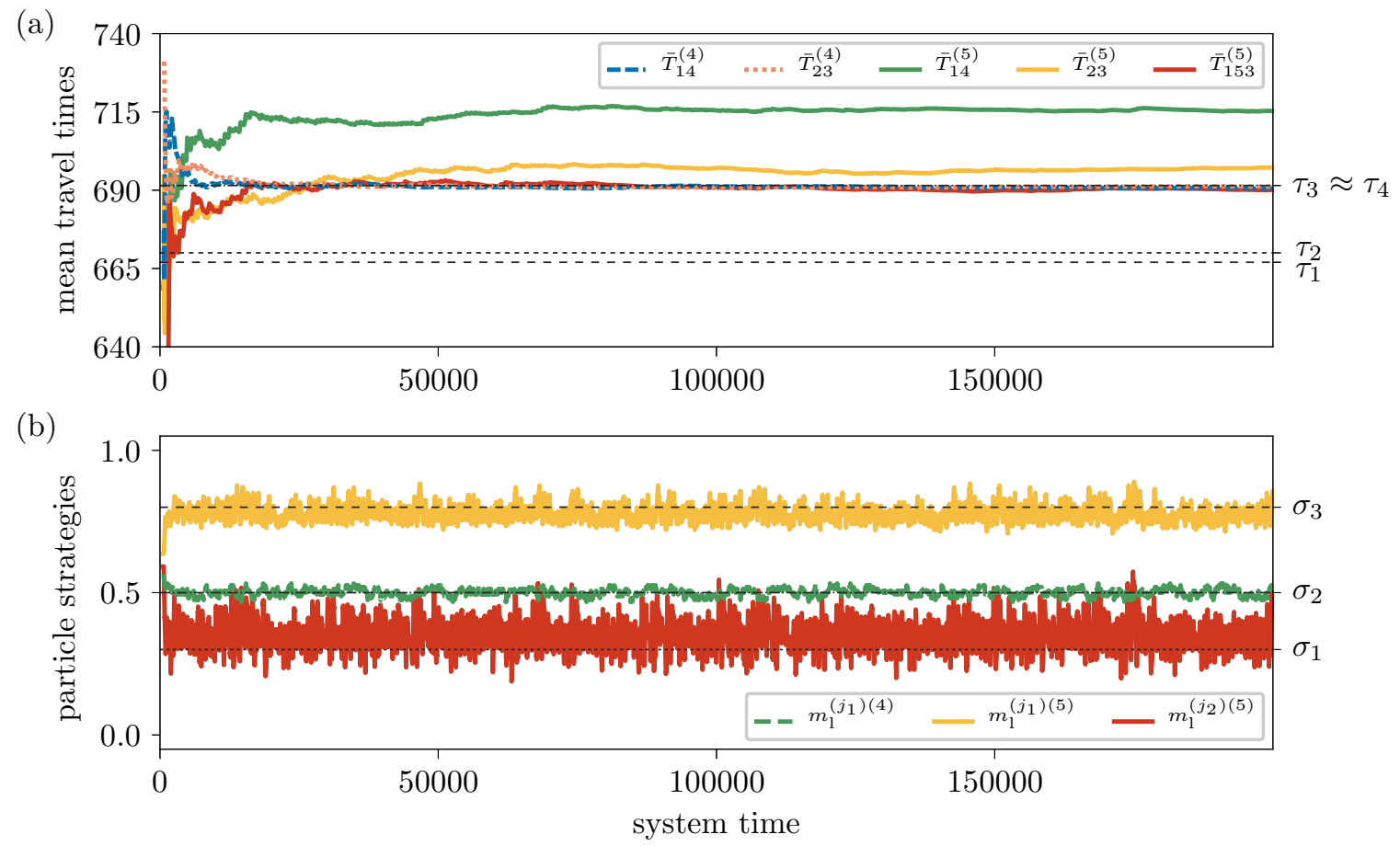

Figure 5. Results for state $\star 2$ if public predictive information is provided. As seen in (a), the mean travel times of both routes in the 4link system coincide with those from the expectations. In the 5link system, all three routes have higher mean travel times than expected in the pure and mixed 5link user optima. The travel times are also higher than those in the 4link. The expected travel times in the pure and mixed user optima found by externally tuning the strategies are shown by the dotted lines with $T_{\text {max }, \text { puo }}^{(4)}=\tau_{3}, T_{\max , \text { muo }}^{(4)}=\tau_{4}, T_{\text {max }, \text { puo }}^{(5)}=\tau_{2}, T_{\text {max }, \text { muo }}^{(5)}=\tau_{1}$. Part (b) shows that in the 4link both routes are, as expected, used by equal amounts of agents. In the 5link system fluctuations around the expected user optima are observed. The strategies realizing the user optima by externally tuning the strategies are given for comparison with $n_{1, \text { puo }}^{\left(j_{1}\right)(4)}=\gamma_{\text {muo }}^{(4)}=\sigma_{2},\left(n_{1, \text { puo }}^{\left(j_{1}\right)(5)}, n_{1, \text { puo }}^{\left(j_{2}\right)(5)}\right)=\left(\gamma_{\text {muo }}^{(5)}, \delta_{\text {muo }}^{(5)}\right)=\left(\sigma_{3}, \sigma_{1}\right)$. Judging from the mean travel time values, a Braess state instead of the expected " $E_{5}$ optimal" state is observed.

Even if fluctuations around the 4link user optimum are also small in state $\star 4$, the travel times of both routes equalize at a slightly higher value than expected (Fig. 7 (a)). This is a consequence of jamming effects in front of $j_{4}$ which play a larger role at higher densities. In the 5link network with externally tuned particles only a pure user optimum exists (see Appendix B). In the system with externally tuned turning probabilities fluctuating domain walls are observed at such high densities and thus no (short term) user optimum exists [19]. The route choice algorithm drives the system close to the pure user optimum. The resulting $m_{1}^{\left(j_{1}\right)(5)}$ and $m_{1}^{\left(j_{2}\right)(5)}$ are slightly different from the expected $\left(n_{1, \text { puo }}^{\left(j_{1}\right)(5)}, n_{1, \text { puo }}^{\left(j_{2}\right)(5)}\right)$ in the pure user optimum. Furthermore they fluctuate (Fig. 7 (b)). Thus the travel times of the three routes are not equal. Routes 23 and 153 have higher travel times than the routes of the 4link system (Fig. 7 (b)), which can be interpreted as a kind of Braess 

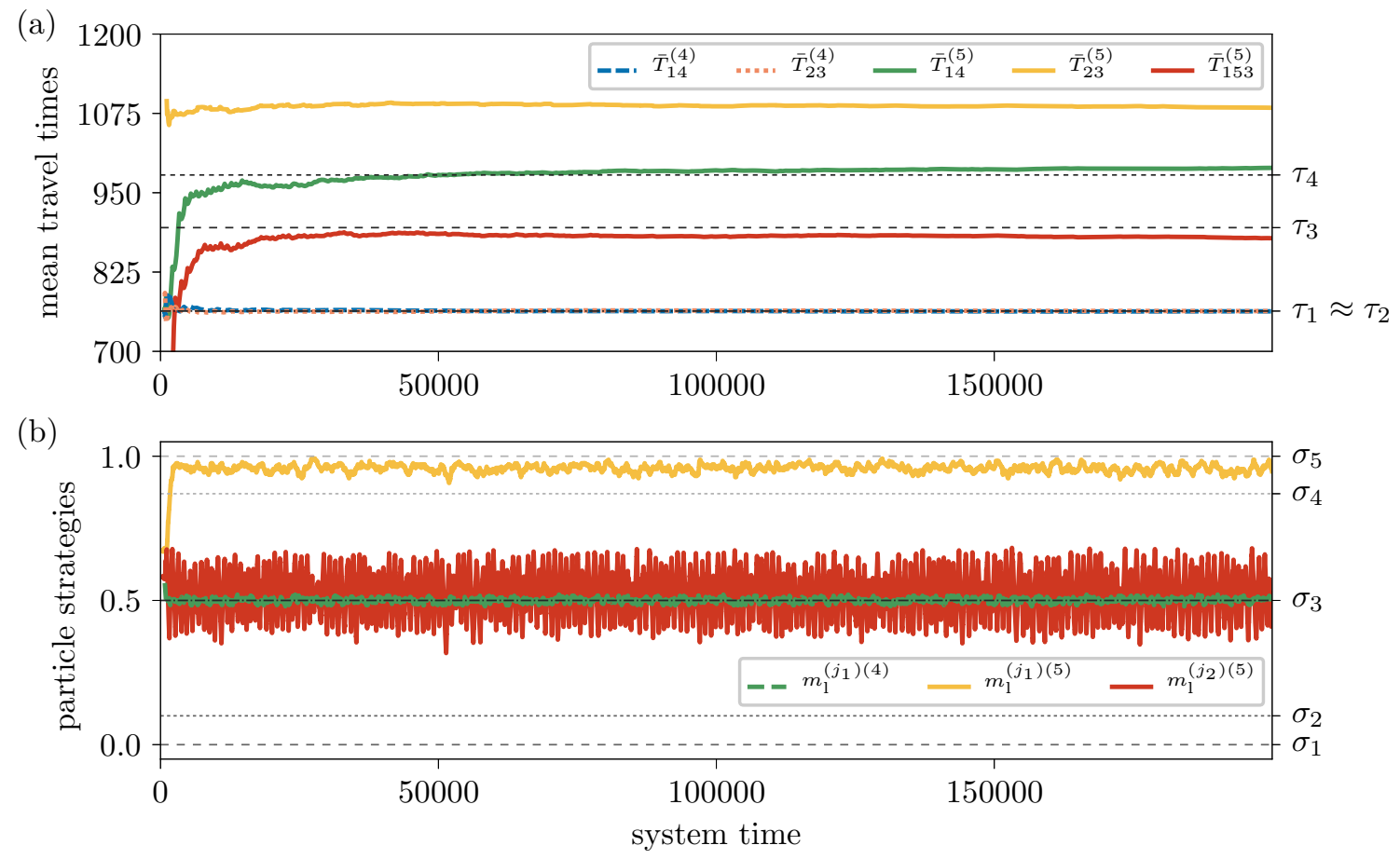

Figure 6. Results for state $\star 3$ if public predictive information is provided. As seen in (a), the mean travel times of both routes in the 4link system coincide with those from the expectations. In the 5link system, all three routes have unequal travel times, all higher than those in the 4link. Travel times of route 14 and 153 are similar and close to those expected in the 5link user optima. The expected travel times in the pure and mixed user optima found by externally tuning the strategies are shown by the dotted lines with $T_{\max , \text { puo }}^{(4)}=\tau_{2}, T_{\max , \operatorname{muo}}^{(4)}=\tau_{1}, T_{\max , \operatorname{puo}(\mathrm{i})}^{(5)}=T_{\max , \mathrm{puo}(\mathrm{ii})}^{(5)}=\tau_{4}$, $T_{\max , \text { muo }}^{(5)}=\tau_{3}$. Part (b) shows that in the 4link both routes are, as expected, used by equal amounts of particles. In the 5link system strong fluctuations around one of the three theoretically accessible user optima, i.e. around the pure user optimum puo(ii), are observed. Route 23 is not used by many particles. The strategies realizing the user optima by externally tuning the strategies are given for comparison with $n_{1, \text { puo }}^{\left(j_{1}\right)(4)}=\gamma_{\text {muo }}^{(4)}=\sigma_{3},\left(n_{1, \text { puo }(\mathrm{i})}^{\left(j_{1}\right)(5)}, n_{1, \text { puo }(\mathrm{i})}^{\left(j_{2}\right)(5)}\right)=\left(\sigma_{3}, \sigma_{1}\right),\left(n_{1, \text { puo }(\mathrm{ii})}^{\left(j_{1}\right)(5)}, n_{1, \text { puo }(\mathrm{ii})}^{\left(j_{2}\right)(5)}\right)=\left(\sigma_{5}, \sigma_{3}\right)$, $\left(\gamma_{\text {muo }}^{(5)}, \delta_{\text {muo }}^{(4)}\right)=\left(\sigma_{4}, \sigma_{2}\right)$. The "Braess 1 " state is realized on average.

behaviour.

One can conclude that the route choice based on public predictive information typically drives the system into user optima in the 4link systems. This is not surprising if one remembers how the predicted travel times are calculated (see Eqs. (86)-(10)): since the 4 link system is symmetric and the pure user optima are always given for an equal distribution onto both routes, the algorithm which counts the numbers of particles for its travel time predictions will always realize such user optima.

In the 5link networks user optima are not always realized since road 5 breaks the symmetry of the 4link network. In the 5link network, the public predictive information realizes user optima at low global densities. This is the case since in this density regime 

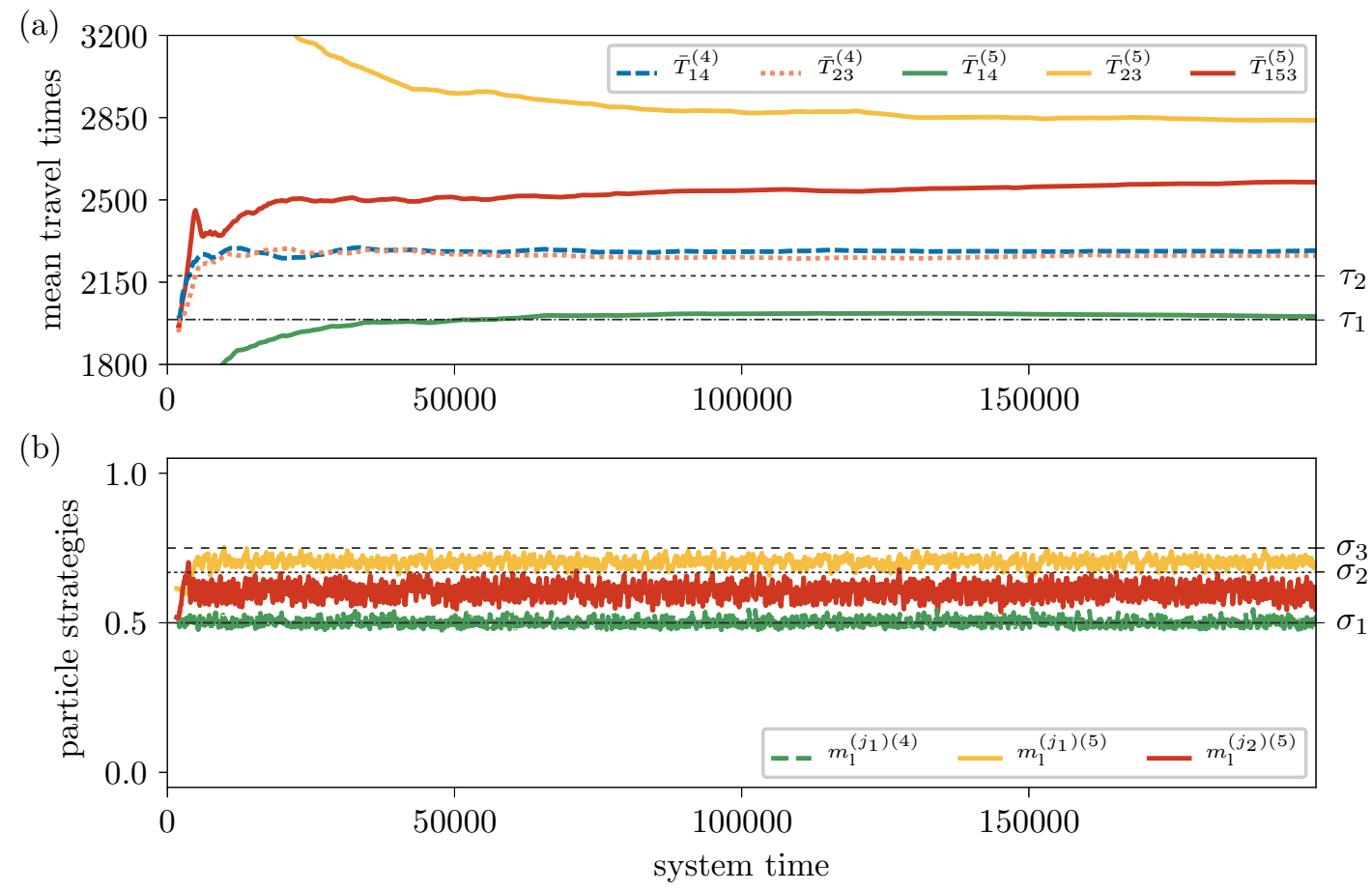

Figure 7. Results for state $\star 4$ if public predictive information is provided. As seen in (a), the mean travel times of both routes in the 4link system equalize, but at a slightly higher value than the expectations. In the 5link system, all three routes have unequal travel times, route 23 and 153 higher than those in the 4link, route 14 lower. The expected travel times in the pure and mixed user optima found by externally tuning the strategies are shown by the dotted lines with $T_{\max , \text { puo }}^{(4)}=\tau_{1}, T_{\max , \text { puo }}^{(5)}=\tau_{2}$. Part (b) shows that in the 4link both routes are, as expected, used by equal amounts of agents. In the 5link system strong fluctuations around the theoretically accessible pure user optima are observed. The strategies realizing the user optima by externally tuning the strategies are given for comparison with $n_{1, \text { puo }}^{\left(j_{1}\right)(4)}=\sigma_{1},\left(n_{1, \text { puo }}^{\left(j_{1}\right)(5)}, n_{1, \text { puo }}^{\left(j_{2}\right)(5)}\right)=\left(\sigma_{3}, \sigma_{2}\right)$.

the correlations between the roads do not influence the route travel times strongly. At higher densities the correlations become more important leading to traffic jams near junction sites. Here the travel time predictions become less accurate, leading to fluctuations around the user optima. This can be seen in Appendix C, where the accuracy of the predicted travel times is shown.

\subsection{Personal historical information.}

Results for the case of personal historical information are shown in Figs. 811 for the four different states $\star 1$ to $\star 4$. In all parts (a) and (b) of these figures four vertical lines are shown. The two lines in brighter and darker grey correspond to the two relaxation times of the 4link and 5link systems, respectively. The line further to the left indicates the system time at which all agents have gathered at least one travel time experience for each route. The line further to the right indicates the system time at which the memory 
capacities of all agents is full. The recording of the evolution of the mean travel time starts once the relaxation process is finished.

Fig. 8 shows results for state $\star 1$. The user optimum in the 4 link system is reached with small remaining fluctuations around the user optimum (Fig. 8 (b)). The travel times of both routes in the 4link equalize at the expected values (Fig. 8 (a)). In the

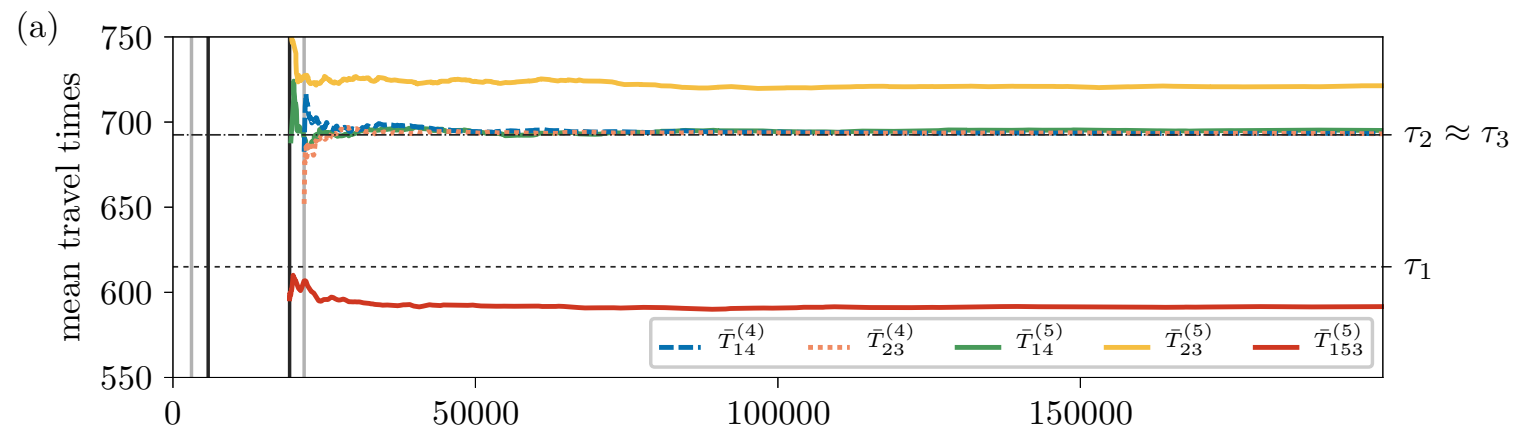

(b)

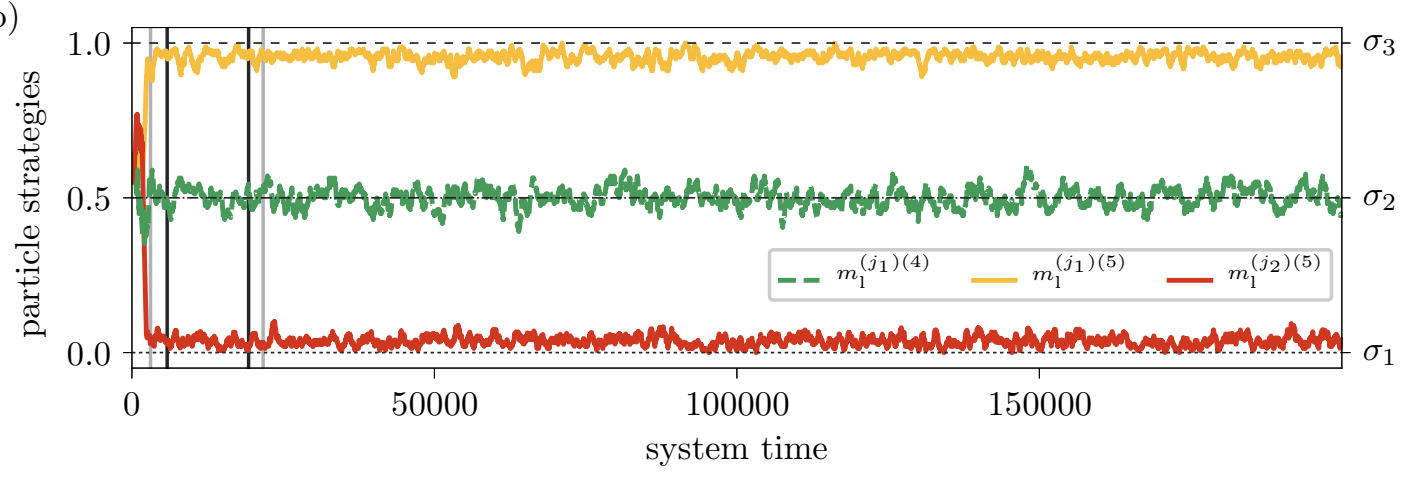

Figure 8. Results for state $\star 1$ if personal historical information is provided. As seen in (a), the mean travel times on the routes in the 4link system equalize at the expected values. In the 5link system, the travel time on route 153 is slightly lower than expected. The expected travel times in the pure and mixed user optima found by externally tuning the strategies are shown by the dotted lines with $T_{\max \text {,puo }}^{(4)}=\tau_{2}$, $T_{\text {max }, \text { muo }}^{(4)}=\tau_{3}, T_{\max , \text { puo }}^{(5)}=T_{\max , \text { muo }}^{(5)}=\tau_{1}$. Part (b) shows that in the 4link and 5link networks, the strategies develop as expected in the theoretically accessible user optima. In the 5link, route 153 is used almost exclusively. The strategies realizing the user optima by externally tuning the strategies are given for comparison with $n_{1, \text { puo }}^{\left(j_{1}\right)(4)}=\gamma_{\text {muo }}^{(4)}=\sigma_{2},\left(n_{1, \text { puo }}^{\left(j_{1}\right)(5)}, n_{1, \text { puo }}^{\left(j_{2}\right)(5)}\right)=\left(\gamma_{\text {muo }}^{(5)}, \delta_{\text {muo }}^{(5)}\right)=\left(\sigma_{3}, \sigma_{1}\right)$. The " $E_{5}$ optimal, all 153 " state is realized.

5link system the user optimum is also realized with some remaining fluctuations. Almost all agents use route 153 (Fig. 8 (b)) which has a lower travel time than the other two (almost unused) routes and also a lower travel time than the routes in the 4link system.

The results for state $\star 2$ are shown in Fig. 9. Both in the 4link and 5link system the user optimum is reached with some minor fluctuations (Fig. 9 (b)), as in state $\star 1$. The mean travel times of all three routes in the 5link system are almost equal to the expected values and lower than those of the 4link system (Fig. 9 (a)). In contrast to the algorithm with public predictive information where the 5link travel times were higher 

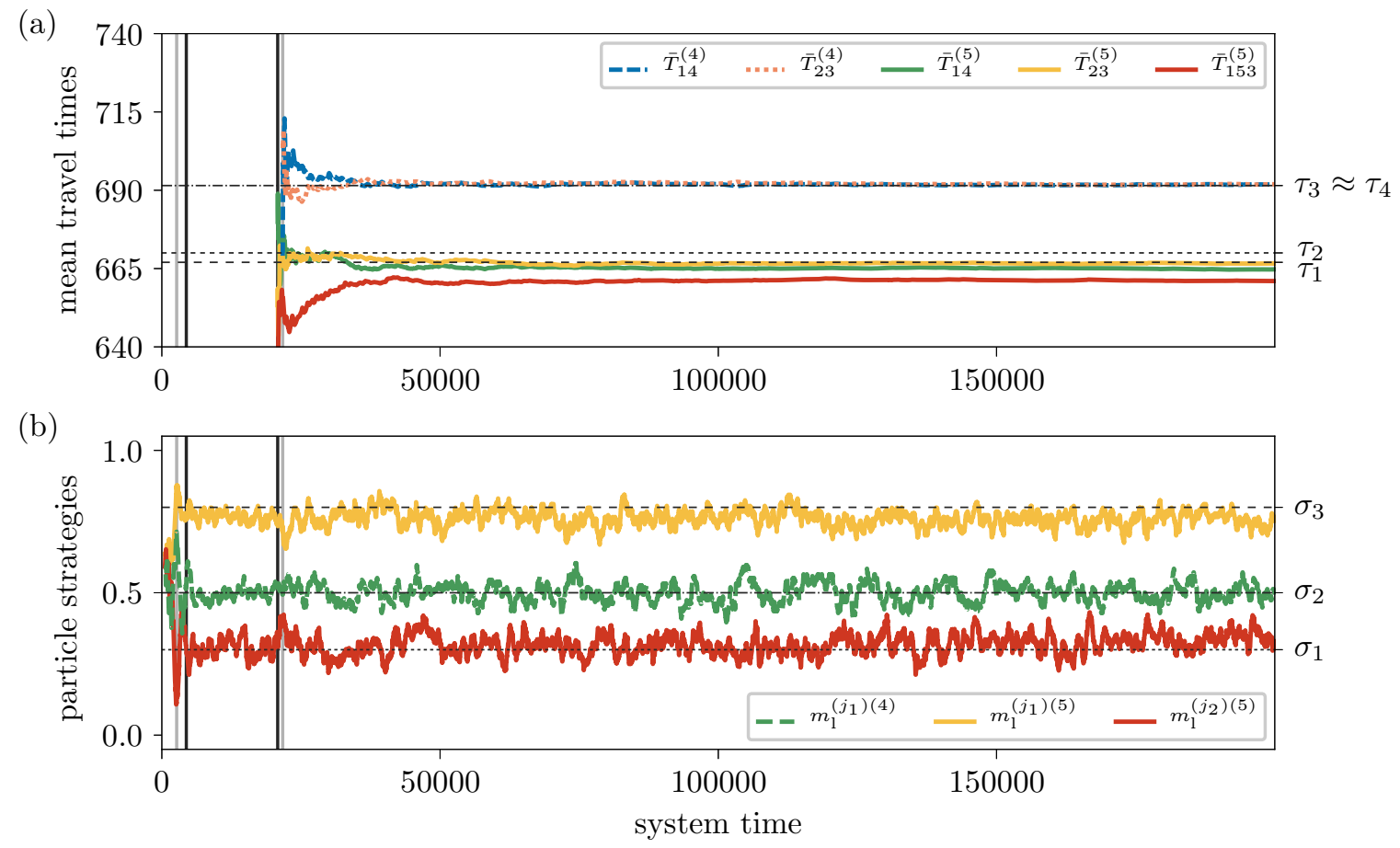

Figure 9. Results for state $\star 2$ if personal historical information is provided. As seen in (a), the mean travel times on the routes in the 4link and 5link systems equalize at the expected values. The expected travel times in the pure and mixed user optima found by externally tuning the strategies are shown by the dotted lines with $T_{\max , \text { puo }}^{(4)}=\tau_{3}$, $T_{\max , \text { muo }}^{(4)}=\tau_{4}, T_{\max , \text { puo }}^{(5)}=\tau_{2}, T_{\max , \text { muo }}^{(5)}=\tau_{1}$. Part (b) shows that in the 4link and 5link networks, the strategies develop as expected in the theoretically accessible user optima. The strategies realizing the user optima by externally tuning the strategies are given for comparison with $n_{1, \text { puo }}^{\left(j_{1}\right)(4)}=\gamma_{\text {muo }}^{(4)}=\sigma_{2},\left(n_{1, \text { puo }}^{\left(j_{1}\right)(5)}, n_{1, \text { puo }}^{\left(j_{2}\right)(5)}\right)=\left(\gamma_{\text {muo }}^{(5)}, \delta_{\text {muo }}^{(5)}\right)=\left(\sigma_{3}, \sigma_{1}\right)$. The " $E_{5}$ optimal" state is realized.

than the 4link's and the system thus showed Braess behaviour (Fig. 6 (b)), here the expected " $E_{5}$ optimal" state is realized.

Fig. 10 shows results for state $\star 3$. As in the previous states $\star 1$ and $\star 2$ the 4 link user optimum is reached with some minor fluctuations. In the 5link system, the pure user optimum puo(ii) (see Appendix B) is realized by the algorithm. Apart from some minor fluctuations, approximately $M / 2$ agents use routes 14 and 153, respectively. The travel times of these routes equalize at a travel time below that of the almost unused route 23 and above the travel times in the 4 link system. The 5link user optimum is reached in a more stable manner than in the system with public predictive information (see Fig. 6).

Fig. 11] shows results for the case of personal historical information in state $\star 4$. One can see that in this case a long relaxation process is needed. This is due to the high global density: as all agents want to gather travel time experiences for all three routes in the beginning, this leads to routes getting blocked. The blockages are not permanent gridlocks since agents will re-decide their route choices if they have to wait very long 

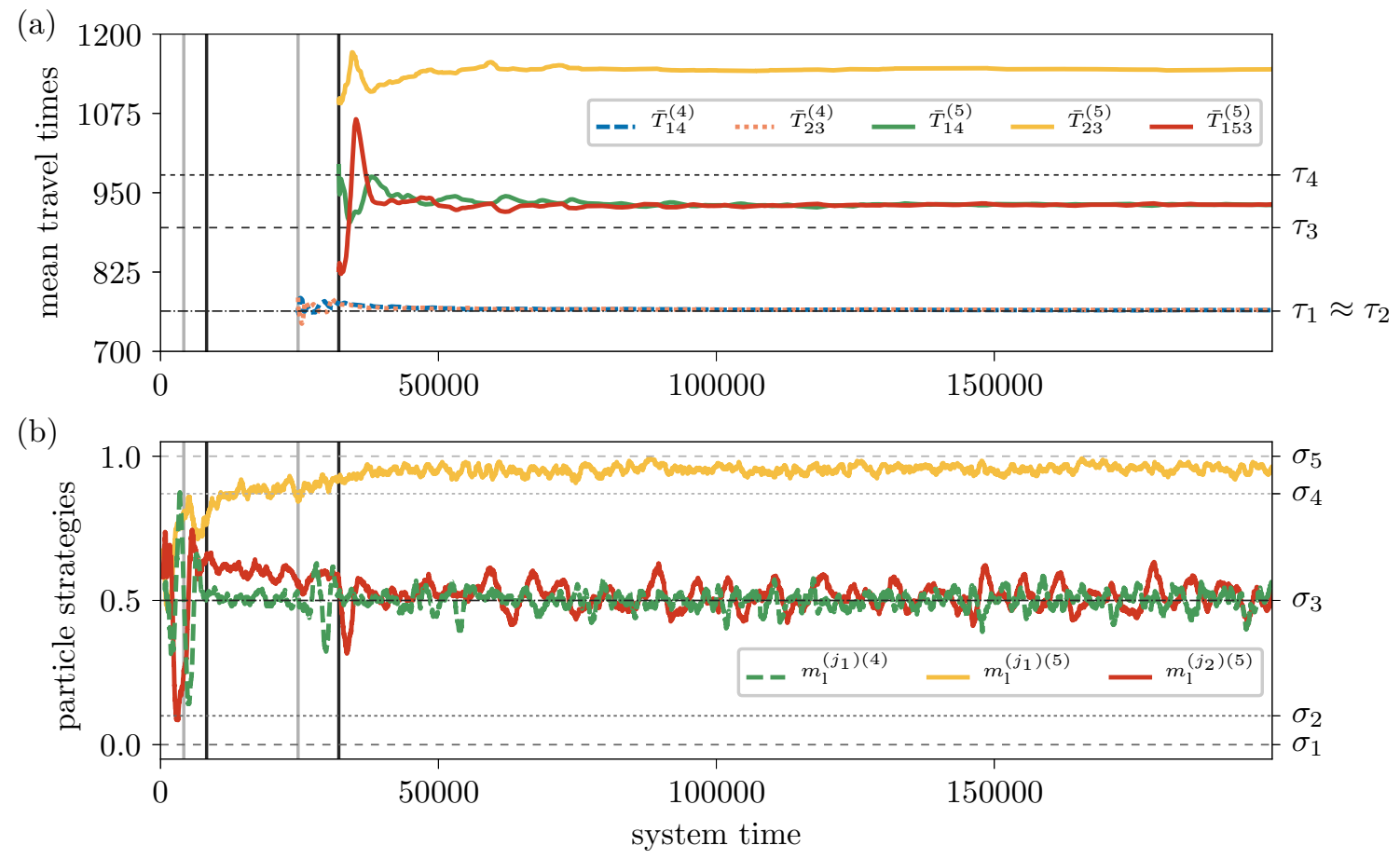

Figure 10. Results for state $\star 3$ if personal historical information is provided. As seen in (a), the mean travel times of the routes in the 4link system equalize at the expected values. In the 5link system, the mean travel times of routes 14 and 153 equalize at a value lower than that of route 23 , but higher than those of the 4link system. The expected travel times in the pure and mixed user optima found by externally tuning the strategies are shown by the dotted lines with $T_{\max , \text { puo }}^{(4)}=\tau_{2}$, $T_{\max , \text { muo }}^{(4)}=\tau_{1}, T_{\max , \text { puo(i) }}^{(5)}=T_{\max , \text { puo(ii) }}^{(5)}=\tau_{4}, T_{\text {max }, \text { muo }}^{(5)}=\tau_{3}$. Part (b) shows that in the 4 link the strategies develop as expected in the theoretically accessible user optima. In the 5link system, the pure user optimum puo(ii) is approached with some small fluctuations. In the 5link system, routes 14 and 153 are used by approximately half the agents each, while route 23 is almost not used at all. The strategies realizing the user optima by externally tuning the strategies are given for comparison with $n_{1, \text { puo }}^{\left(j_{1}\right)(4)}=\gamma_{\text {muo }}^{(4)}=\sigma_{3},\left(n_{1, \text { puo(i) }}^{\left(j_{1}\right)(5)}, n_{1, \text { puo(i) }}^{\left(j_{2}\right)(5)}\right)=\left(\sigma_{3}, \sigma_{1}\right),\left(n_{1, \text { puo(ii) }}^{\left(j_{1}\right)(5)}, n_{1, \text { puo(ii) }}^{\left(j_{2}\right)(5)}\right)=\left(\sigma_{5}, \sigma_{3}\right)$, $\left(\gamma_{\text {muo }}^{(5)}, \delta_{\text {muo }}^{(4)}\right)=\left(\sigma_{4}, \sigma_{2}\right)$. The "Braess 1" state is realized.

at junctions $j_{1}$ or $j_{2}$. Nevertheless it takes quite long until the whole system is relaxed. Once it is relaxed it stabilises quickly. As can be seen in Fig. 11](b), in the 4link system the user optimum is reached with larger fluctuations around the expected state than for states $\star 1$ to $\star 3$. The effect of these larger fluctuations can also be seen in the mean travel times of the two routes in the 4link: Fig. 11 (a) shows that the travel times' mean values only equalize after a relatively long time. The 5link systems' user optimum is also realized on average. Fluctuations around the pure user optimum persist (Fig. 11 (b)). The mean travel times of the three routes are almost equal to the expected value of the pure user optimum, above the travel times of the 4link system. Thus, here also a Braess state is realized. 

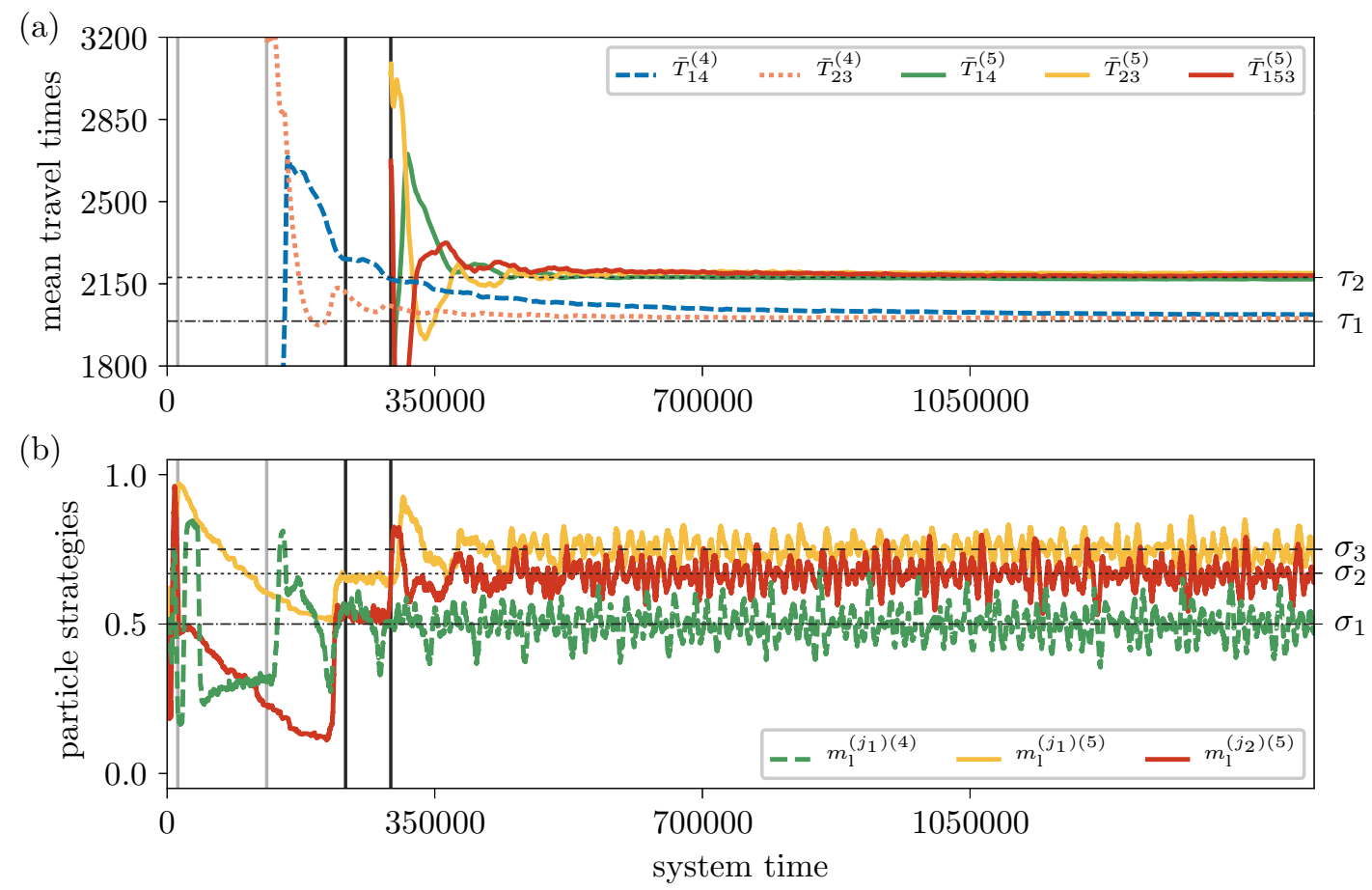

Figure 11. Results for state $\star 4$ if personal historical information is provided. In this state with higher global density than states $\star 1$ to $\star 3$ a longer relaxation time is needed, which is why a longer system time period is plotted as compared to Figs. 4 to 10. As seen in (a), the mean travel times of the routes in the 4link and 5link systems equalize at the expected values. The expected travel times in the pure and mixed user optima found by externally tuning the strategies are shown by the dotted lines with $T_{\text {max } \text {,puo }}^{(4)}=\tau_{1}, T_{\text {max } \text { puo }}^{(5)}=\tau_{2}$. Part (b) shows that in the 4link and 5link the strategies develop as expected in the theoretically accessible user optima. The strategies realizing the user optima by externally tuning the strategies are given for comparison with $n_{1, \text { puo }}^{\left(j_{1}\right)(4)}=\sigma_{1},\left(n_{1, \text { puo }}^{\left(j_{1}\right)(5)}, n_{\mathrm{l}, \text { puo }}^{\left(j_{2}\right)(5)}\right)=\left(\sigma_{3}, \sigma_{2}\right)$. The "Braess 1" state, which for externally tuned route choices only exists with fixed route choices, is realized.

One can conclude that user optima of both the 4link and the 5link systems are realized in all four states by the algorithm with personal historical information.

\section{Conclusions}

For drivers using public predictive information, user optima are realized at low global densities. At higher global densities the user optima are still reached on average. The kind of predictive information that we implemented depends on the current positions of all agents in the system. It employs an approximation formula for the travel times based on these positions. This kind of information is similar to that used in modern smartphone apps which rely on crowdsourced data. The fact that the expected user optima are realized, also in the Braess states, is a strong hint at the importance of the paradox in modern traffic networks. As already proposed by previous observations of 
real world data [36, 37], smartphone apps seem to support the realization of user optima in road networks.

In scenarios where drivers utilize only their own memories of travel times we could also show that the user optima of the four test states are realized. Similar behaviour in a two-route system without correlations and dynamics similar to TASEP and a similar type of personal historical information was observed in [62], indicating that this finding is rather general. We could show that the user optima are also realized in networks with correlations: both in our 4link and 5link network the expected user optima are realized. This further strengthens the importance of the paradox, since the reliance on personal historical information is likely relevant in many commuter's scenarios.

In future research it would be interesting to consider combinations of the latter two types of information and see if drivers depending on different types of information influence each other and the system as a whole. Are user optima also realized in this case?

\section{Acknowledgements}

Financial support by Deutsche Forschungsgemeinschaft (DFG), Germany under grant SCHA 636/8-2 is gratefully acknowledged. Also support by the Bonn-Cologne Graduate School of Physics and Astronomy (BCGS) is acknowledged. Monte Carlo simulations were carried out on the CHEOPS (Cologne High Efficiency Operating Platform for Science) cluster of the RRZK (University of Cologne).

\section{Appendix A. Mixed user optima in Braess' original example}

As described in Sec. 2.2, the Braess paradox is also observed in Braess' original model if users choose their routes according to mixed strategies. Let $p_{14}, p_{23}$ and $p_{153}$ be the probabilities with which all users choose routes 14, 23 and 153, respectively. The probabilities are subject to $p_{14}+p_{23}=1$ or $p_{14}+p_{23}+p_{153}=1$ for the 4link and 5link systems, respectively.

In the 4link system, for mixed strategies (ms) the expectation values, denoted by $\left\langle T_{i, \mathrm{~ms}}^{(4)}\right\rangle$, of the travel times on the routes 14 and 23 are

$$
\begin{aligned}
& \left\langle T_{14, \mathrm{~ms}}^{(4)}\right\rangle=50+\left(1+p_{14} \cdot(N-1)\right) \cdot 11 \\
& \left\langle T_{23, \mathrm{~ms}}^{(4)}\right\rangle=50+\left(1+p_{23} \cdot(N-1)\right) \cdot 11
\end{aligned}
$$

for each car.

For $N=6$ a mixed user optimum state (muo) is found for $p_{14}=p_{23}=1 / 2$ with a travel time expectation value of $\left\langle T_{14 \text {,muo }}^{(4)}\right\rangle=\left\langle T_{23, \text { muo }}^{(4)}\right\rangle=88.5$.

In the system with the new road, the expectation values of the travel times on the three routes are

$$
\begin{aligned}
& \left\langle T_{14, \mathrm{~ms}}^{(5)}\right\rangle=\left(1+\left(p_{14}+p_{153}\right)(N-1)\right) \cdot 10+50+1+p_{14}(N-1) \\
& \left\langle T_{23, \mathrm{~ms}}^{(5)}\right\rangle=\left(1+\left(p_{23}+p_{153}\right)(N-1)\right) \cdot 10+50+1+p_{23}(N-1)
\end{aligned}
$$




$$
\left\langle T_{153, \mathrm{~ms}}^{(5)}\right\rangle=\left(2+\left(p_{14}+p_{23}+2 p_{153}\right)(N-1)\right) \cdot 10+10+1+p_{153}(N+\mathrm{A} .5)
$$

Here a mixed user optimum is given for $p_{14}=p_{23}=5 / 13$ and $p_{153}=3 / 13$ with travel time values $\left\langle T_{14 \text {,muo }}^{(5)}\right\rangle=\left\langle T_{23 \text {,muo }}^{(5)}\right\rangle=\left\langle T_{153 \text {, muo }}^{(5)}\right\rangle=93.6923$.

For the case of mixed strategies the expected user optimum travel times are also higher in the 5link system than in the 4link system, i.e. the paradox occurs also with mixed strategies.

This example shows that the average number of cars on a specific route in the mixed user optimum does not have to correspond to the (integer) number of cars on that route in the pure user optimum: in the 5link system, the pure user optimum is for $N=6$ given by distributing the users equally on the three routes. The mixed equilibrium is not achieved by all users choosing the routes with equal probability!

\section{Appendix B. The test states}

The parameter sets for which the algorithm was tested are marked in Figs. 3 (a) and (b). The corresponding travel time values that are expected in the existing pure and mixed user optima (as found previously in [19] and [20]) are given in the following. See [19, 20] as well for the naming scheme for the different states.

All these states are boundedly rational user optima [34]: in [19, 20] we found the user optima of the systems by tuning the decisions of the particles externally (either the $N_{14}, N_{23}, N_{153}$ for fixed personal strategies or the $\gamma, \delta$ for turning probabilities) and states for which $\Delta T=\left|T_{14}-T_{23}\right|+\left|T_{14}-T_{153}\right|+\left|T_{23}-T_{153}\right|<100$ were considered to be user optima. This means that the travel times on the three routes are not necessarily exactly equal but are reasonably close to each other and we thus consider the states to be user optima. Since the travel times are not necessarily exactly equal, we give here the maximum travel time observed in those states as the reference time, which is used as a comparison for the system with intelligently deciding particles.

state $\star 1$ : has the parameters $L_{5}=97$ and $M=156$ which correspond to $\hat{L}_{153} / \hat{L}_{14}=$ $0.5, \rho_{\text {global }}^{(4)} \approx 0.13, \rho_{\text {global }}^{(5)}=0.12$. For externally tuned particles this is an " $E_{5}$ optimal, all 153" state both for fixed personal strategies and for turning probabilities.

In the 4link system

- a pure user optimum is given for $N_{14 \text {,puo }}^{(4)}=N_{23 \text {,puo }}^{(4)}=78$ which corresponds to $n_{\mathrm{l}, \text { puo }}^{\left(j_{1}\right)(4)}=0.5$ with $T_{\text {max }, \text { puo }}^{(4)} \approx 692$ and $\Delta T_{\text {puo }}^{(4)} \rightarrow 0$.

- a mixed user optimum is given for $\gamma_{\text {muo }}^{(4)}=0.5$ with $T_{\text {max,muo }}^{(4)} \approx 693$ and $\Delta T_{\text {muo }}^{(4)} \rightarrow 0$.

In the 5link system

- a pure user optimum is given for $N_{14 \text {,puo }}^{(5)}=N_{23 \text {,puo }}^{(5)}=0$ and $N_{153 \text {,puo }}^{(5)}=156$ which corresponds to $n_{\mathrm{l}, \text { puo }}^{\left(j_{1}\right)(5)}=1.0$ and $n_{\mathrm{l}, \text { puo }}^{\left(j_{2}\right)(5)}=0.0$ with $T_{\text {max,puo }}^{(5)} \approx 615$ and $\Delta T_{\text {puo }}^{(5)}=0$.

- a mixed user optimum is given for $\gamma_{\text {muo }}^{(5)}=1.0$ and $\delta_{\text {muo }}^{(5)}=0.0$ with $T_{\text {max,muo }}^{(5)} \approx 615$ and $\Delta T_{\text {muo }}^{(5)}=0$. 
state $\star 2:$ has the parameters $L_{5}=339$ and $M=154$ which correspond to $\hat{L}_{153} / \hat{L}_{14}=$ $0.9, \rho_{\text {global }}^{(4)} \approx 0.13, \rho_{\text {global }}^{(5)}=0.1$. For externally tuned particles this is an " $E_{5}$ optimal" state both for fixed personal strategies and for turning probabilities.

In the 4link system

- a pure user optimum is given for $N_{14 \text {,puo }}^{(4)}=N_{23 \text {,puo }}^{(4)}=77$ which corresponds to $n_{1, \text { puo }}^{\left(j_{1}\right)(4)}=0.5$ with $T_{\text {max } \text {,puo }}^{(4)} \approx 691$ and $\Delta T_{\text {puo }}^{(4)} \rightarrow 0$.

- a mixed user optimum is given for $\gamma_{\text {muo }}^{(4)}=0.5$ with $T_{\text {max }, \text { muo }}^{(4)} \approx 692$ and $\Delta T_{\text {muo }}^{(4)} \rightarrow 0$.

In the 5link system

- a pure user optimum is given for $N_{14, \text { puo }}^{(5)}=36, N_{23 \text {,puo }}^{(5)}=30$ and $N_{153 \text {,puo }}^{(5)}=88$ which corresponds to $n_{1, \text { puo }}^{\left(j_{1}\right)(5)} \approx 0.8$ and $n_{1, \text { puo }}^{\left(j_{2}\right)(5)} \approx 0.3$ with $T_{\text {max,puo }}^{(5)} \approx 670$ and $\Delta T_{\text {puo }}^{(5)} \approx 22$.

- a mixed user optimum is given for $\gamma_{\text {muo }}^{(5)}=0.8$ and $\delta_{\text {muo }}^{(5)}=0.3$ with $T_{\text {max,muo }}^{(5)} \approx 667$ and $\Delta T_{\text {muo }}^{(5)} \approx 12$.

state $\star 3:$ has the parameters $L_{5}=37$ and $M=248$ which correspond to $\hat{L}_{153} / \hat{L}_{14}=$ $0.4, \rho_{\text {global }}^{(4)} \approx 0.21, \rho_{\text {global }}^{(5)}=0.2$. For externally tuned particles this is a "Braess 1 " state both for fixed personal strategies and for turning probabilities.

In the 4link system

- a pure user optimum is given for $N_{14, \text { puo }}^{(4)}=N_{23 \text {,puo }}^{(4)}=124$ which corresponds to $n_{\mathrm{l}, \text { puo }}^{\left(j_{1}\right)(4)}=0.5$ with $T_{\text {max } \text {,puo }}^{(4)} \approx 764$ and $\Delta T_{\text {puo }}^{(4)} \rightarrow 0$.

- a mixed user optimum is given for $\gamma_{\text {muo }}^{(4)}=0.5$ with $T_{\text {max,muo }}^{(4)} \approx 763$ and $\Delta T_{\text {muo }}^{(4)} \rightarrow 0$.

In the 5link system

- two pure user optimum exist for

(i) $N_{14, \text { puo(i) }}^{(5)}=0, N_{23, \text { puo(i) }}^{(5)}=124$ and $N_{153, \text { puo(i) }}^{(5)}=124$ which corresponds to $n_{1, \text { puo(i) }}^{\left(j_{1}\right)(5)}=0.5$ and $n_{1, \text { puo(i) }}^{\left(j_{2}\right)(5)}=0.0$ with $T_{\max , \text { puo }(\mathrm{i})}^{(5)} \approx 978$ and $\Delta T_{\text {puo(i) }}^{(5)}=10$.

(ii) $N_{14, \text { puo(ii) }}^{(5)}=124, N_{23 \text {,puo(ii) }}^{(5)}=0$ and $N_{153 \text {,puo(ii) }}^{(5)}=124$ which corresponds to $n_{1, \text { puo(ii) }}^{\left(j_{1}\right)(5)}=1.0$ and $n_{1, \text { puo(ii) }}^{\left(j_{2}\right)(5)}=0.5$ with $T_{\max , \text { puo(ii) }}^{(5)} \approx 978$ and $\Delta T_{\text {puo(ii) }}^{(5)}=11$.

- a mixed user optimum is given for $\gamma_{\text {muo }}^{(5)}=0.87$ and $\delta_{\text {muo }}^{(5)}=0.1$ with $T_{\text {max } \text {,muo }}^{(5)} \approx 895$ and $\Delta T_{\text {muo }}^{(5)} \approx 78$.

state $\star 4:$ has the parameters $L_{5}=218$ and $M=712$ which correspond to $\hat{L}_{153} / \hat{L}_{14}=$ $0.7, \rho_{\text {global }}^{(4)} \approx 0.59, \rho_{\text {global }}^{(5)}=0.5$. For externally tuned particles this is an "Braess 1 " state for fixed personal strategies. For turning probabilities no user optima could be found for these parameters.

In the 4link system

- a pure user optimum is given for $N_{14, \text { puo }}^{(4)}=N_{23 \text {,puo }}^{(4)}=356$ which corresponds to $n_{1, \text { puo }}^{\left(j_{1}\right)(4)}=0.5$ with $T_{\text {max,puo }}^{(4)} \approx 1991$ and $\Delta T_{\text {puo }}^{(4)} \rightarrow 0$.

- there is no short term mixed user optimum due to fluctuating domain walls 
In the 5link system

- a pure user optimum is given for $N_{14 \text {,puo }}^{(5)}=357, N_{23, \text { puo }}^{(5)}=178$ and $N_{153 \text {,puo }}^{(5)}=177$ which corresponds to $n_{\mathrm{l} \text {, puo }}^{\left(j_{1}\right)(5)}=0.75$ and $n_{1, \text { puo }}^{\left(j_{2}\right)(5)} \approx 0.67$ with $T_{\text {max,puo }}^{(5)} \approx 2177$ and $\Delta T_{\text {muo }}^{(5)} \approx 24$.

- there is no mixed user optimum due to fluctuating domain walls.

\section{Appendix C. Accuracy of the travel time predictions used for public predictive information}

In this section of the Appendix we discuss the accuracy of the travel time predictions that are provided in the case of public predictive information, i.e. we discuss how accurate

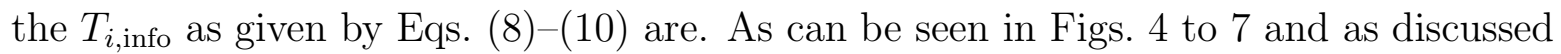
in Section 4, only in state $\star 1$ the the expected user optimum is realized in a stable manner, while in states $\star 2$ to $\star 4$ especially in the 5link systems fluctuations around user optima persist, resulting in (slightly) unequal mean travel times of the routes. In Figs. C1 to C4 we show how accurate the travel time predictions are. For this, the relative error of the $T_{i \text {,info }}$ compared to the actually measured $T_{i}$ are given: the $T_{i \text {,info }}$ of all routes $i$, predicted before starting a new round are saved for each particle. Once an agent finishes a round, the travel time that the agent actually experienced on its chosen route $i\left(T_{i}\right)$ is measured. From this the relative error is computed as $\left(T_{i \text {,info }}-T_{i}\right) / T_{i}$.
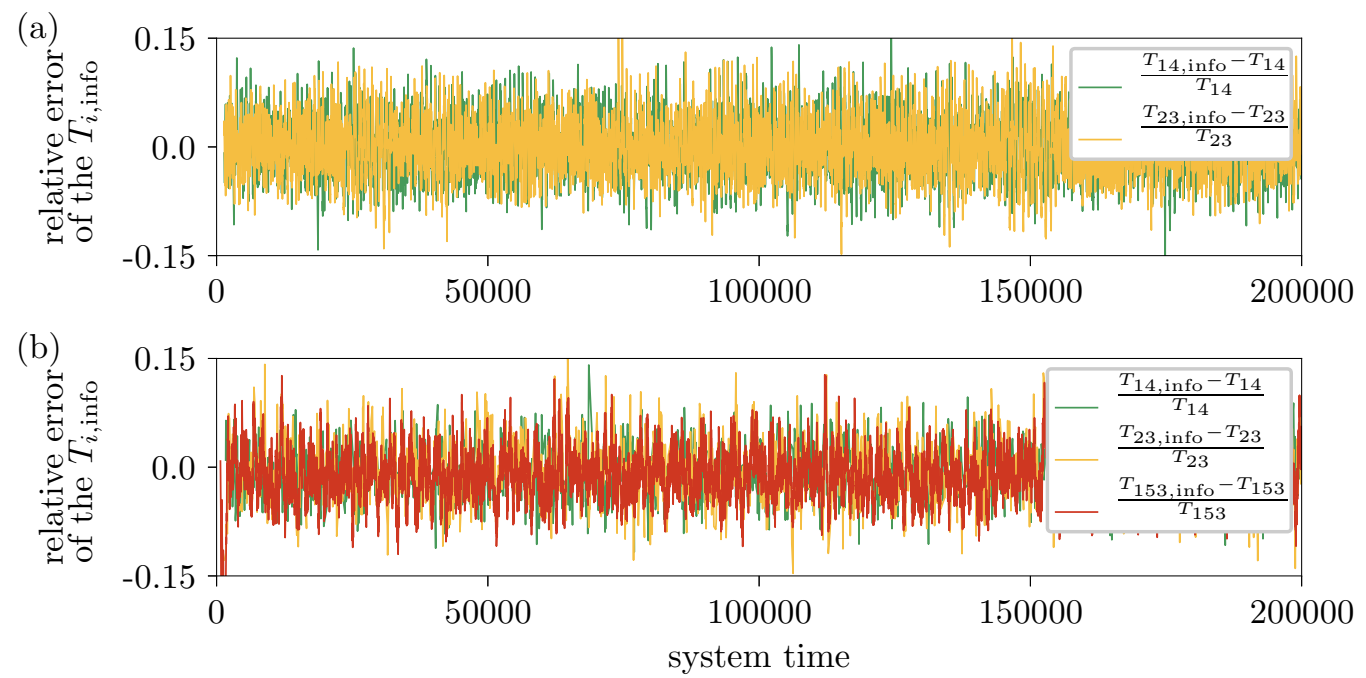

Figure C1. Accuracy of the public predictive travel time predictions $T_{i \text {,info for state }}$ $\star 1$ in (a) the 4link system and (b) the 5link system, corresponding to the data shown in Figure 4 ,

In Fig. C1 it can be seen that in state $\star 1$ the predicted travel times are fairly accurate, as the relative error of the predictions as compared to the actual measured travel times lies beneath $15 \%$ at all times. This is not surprising since the global density 
is very low in that state and thus only small jamming effects and other correlationinduced effects are expected. Judging from Fig. (4 (b) one can furthermore see that in the 5link system almost all particles use route 153. Thus the system is more or less a single periodic TASEP, for which Equation (7) is correct.
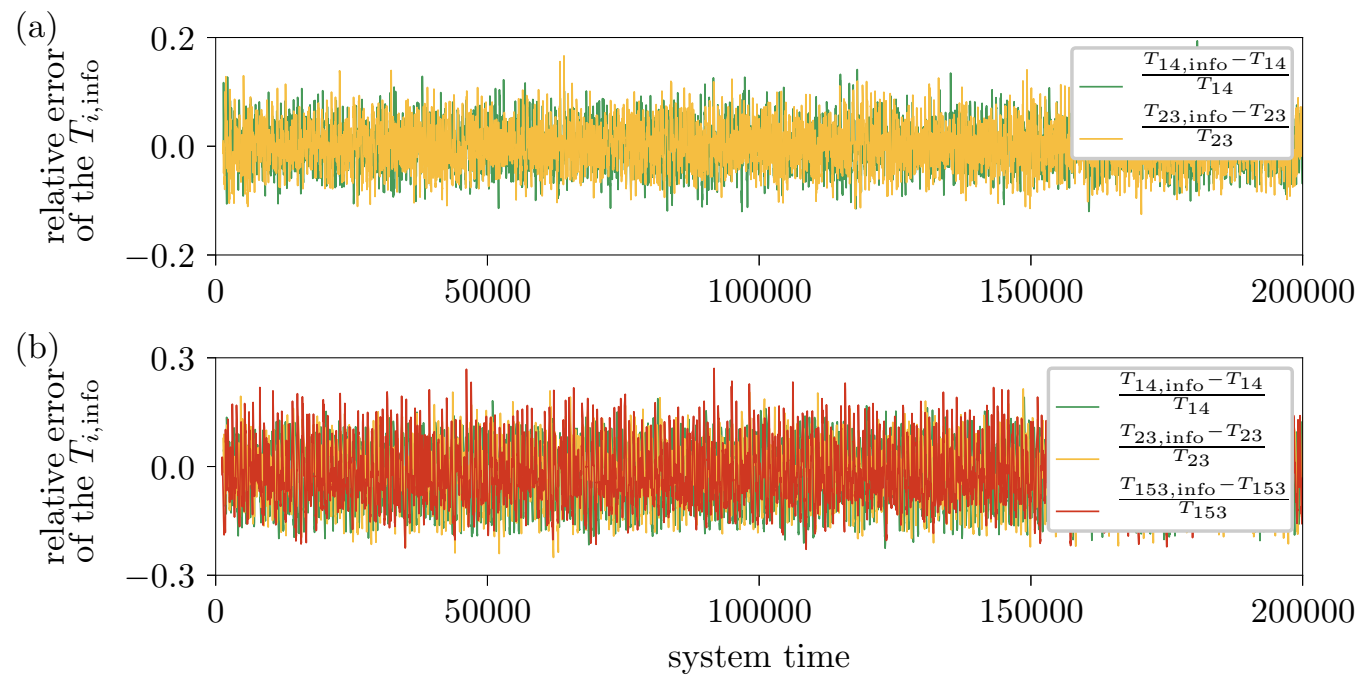

Figure C2. Accuracy of the public predictive travel time predictions $T_{i, \text { info }}$ for state $\star 2$ in (a) the 4link system and (b) the 5link system, corresponding to the data shown in Figure 5 .

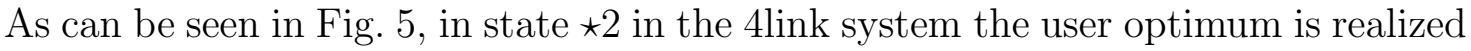
in a stable way. Judging from Fig. C2 (a) we can see that in this case the travel time predictions are also accurate. This is for the same reasons as stated above for the 4link system of state $\star 1$. In the 5link system the user optimum is only reached on average (Fig. (5)). In Fig. C2 (b) it can be seen that the predicted travel times are off for up to almost $\pm 30 \%$.

In state $\star 3$, the predictions in the 4 link system are still fairly accurate, as seen in Fig. C3 (a). Accordingly also the user optimum in the 4link is realized well (Fig. 6). In the 5link system we can see, that the predicted travel times are of by a large degree, especially for route 153, they are at times more than $100 \%$ off. In Fig. C3 (b) one can see, that at times when the travel time on route 153 is predicted too high, the travel time on route 14 is predicted too low and vice versa. This could be the cause for the fluctuations around the user optimum that can be seen in Fig. 6.

In state $\star 4$ the global density has a value of $\rho_{\text {global }}^{(4)} \approx 0.59$ and $\rho_{\text {global }}^{(5)}=0.5$ and is thus much higher than in the three other states. At a higher global density also jamming effects, which are not covered by the simple approximations used for the travel time predictions, become more important. As can be seen in Fig. C4 (a), in the 4link system the travel times are predicted too low. This could be a consequence of neglecting correlations and could be the cause of the slightly higher oscillations around the optimum in the 4link system of state $\star 4$ (Fig. 7 (b)) as compared to the 4 link system of the other 
(a)

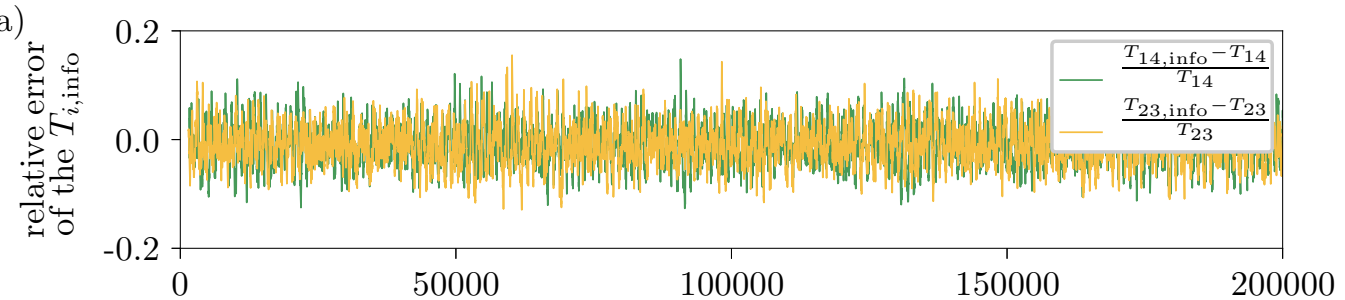

(b)

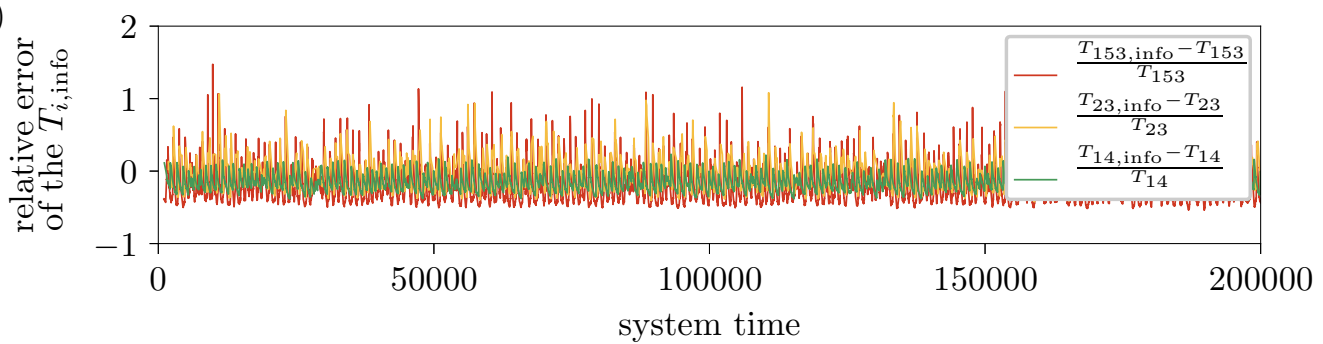

Figure C3. Accuracy of the public predictive travel time predictions $T_{i, \text { info }}$ for state $\star 3$ in (a) the 4link system and (b) the 5link system, corresponding to the data shown in Figure 6 .
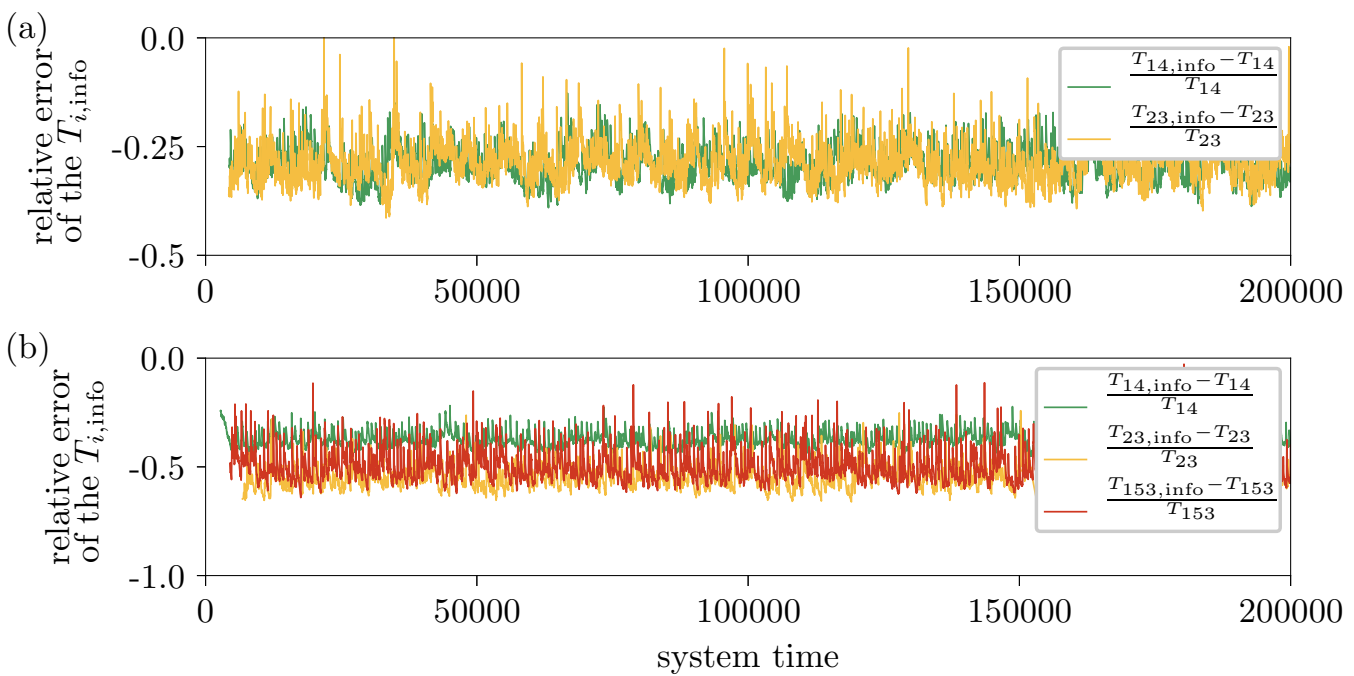

Figure C4. Accuracy of the public predictive travel time predictions $T_{i \text {,info }}$ for state $\star 4$ in (a) the 4link system and (b) the 5link system, corresponding to the data shown in Fig. 7

states and the resulting higher mean travel times than those expected from the user optimum in systems with externally tuned strategies (Fig. 7 (a)). In Fig. C4 (b) one can see that the travel times in the 5link system are also predicted too low. This could also be the reason for the imperfect realization of the 5link's user optimum (Fig. 7). 


\section{References}

[1] Cookson G and Pishue B 2017 INRIX Research, February

[2] TomTom International BV 2017 TomTom Traffic Index (Online, accessed 26September-2018)

[3] United Nations 2018 (Online, accessed 26-September-2018)

[4] Duranton G and Turner M A 2011 American Economic Review 101 2616-52

[5] Braess D 1968 Unternehmensforschung 12258

[6] Braess D, Nagurney A and Wakolbinger T 2005 Transp. Sci. 39446 (English translation of [5])

[7] Steinberg R and Zangwill W 1983 Transp. Sci. 17301

[8] Dafermos S and Nagurney A 1984 Transp. Res. B 18101

[9] Pas E I and Principio S L 1997 Transp. Res. Part B: Methodological 31 265-276

[10] Penchina C and Penchina L 2003 Am. J. Phys. 71(5) 479

[11] Cohen J E and Horowitz P 1991 Nature 352699

[12] Crociani L and Lämmel G 2016 Computer-Aided Civil and Infrastructure Engineering 31 432-448

[13] Case D, Liu Y, Kiss I, Angilella J R and Motter A 2019 Nature 574647

[14] Witthaut D and Timme M 2012 New Journal of Physics 14083036

[15] Tchuisseu E B T, Gomila D, Colet P, Witthaut D, Timme M and Schfer B 2018 New Journal of Physics 20083005

[16] Motter A E and Timme M 2018 Annual Review of Condensed Matter Physics 9 463-484

[17] Wardrop J G 1952 Proceedings of the Institution of Civil Engineers 1 325-362

[18] Stewart N F 1980 Transp. Res. Part A: General 14 81-84

[19] Bittihn S and Schadschneider A 2016 Phys. Rev. E 94(6) 062312

[20] Bittihn S and Schadschneider A 2018 Physica A: Statistical Mechanics and its Applications 507133 - 152

[21] G Kolata December 25, 1990 New York Times URL www.nytimes.com/1990/12/ 25/health/what-if-they-closed-42d-street-and-nobody-noticed.html

[22] Baker L 2009 Scientific American URL https://www.scientificamerican.com/ article/removing-roads-and-traffic-lights/

[23] Vidal J November 2006 The Guardian URL https://www.theguardian.com/ environment/2006/nov/01/society. travelsenvironmentalimpact

[24] Youn H, Gastner M and Jeong H 2007 Phys. Rev. Lett. 101128701

[25] Murchland J D 1970 Transp. Res. 4 391-394

[26] Frank M 1981 Mathematical Programming 20 283-302

[27] Nagurney A 2010 EPL (Europhysics Letters) 9148002 
[28] Parthasarathi P, Levinson D and Hochmair H 2013 PLOS ONE 8 1-13

[29] Chen T Y, Chang H L and Tzeng G H 2001 Transp. Res. Part A: Policy and Practice 35 197-224

[30] Zhu S and Levinson D 2015 PLOS ONE 10 1-18

[31] Rapoport A, Kugler T, Dugar S and Gisches E 2009 Games and Economic Behavior $65538-571$

[32] Selten R, Chmura T, Pitz T, Kube S and Schreckenberg M 2007 Games and Economic Behavior 58394 - 406

[33] Daganzo C F and Sheffi Y 1977 Transp. Sci. 11 253-274

[34] Mahmassani H S and Chang G L 1987 Transp. Sci. 21 89-99

[35] Meneguzzer C and Olivieri A 2013 Procedia-Social and Behavioral Sciences 87 $44-59$

[36] A C Madrigal 2018 The Atlantic (Online, accessed 11-September-2018) URL https://www.theatlantic.com/technology/archive/2018/03/ mapping-apps-and-the-price-of-anarchy/555551/

[37] Cabannes T, Vincentelli M A S, Sundt A, Signargout H, Porter E, Fighiera V, Ugirumurera J and Bayen A M 2018 The impact of GPS-enabled shortest path routing on mobility: a game theoretic approach Tech. rep. Transportation Research Board

[38] Nash J F 1950 Proc. Nat. Acad. Sci. 36 48-49

[39] Schütz G 2000 Phase Transitions and Critical Phenomena 191

[40] Blythe R and Evans M 2007 J. Phys. A 40 R333

[41] Schadschneider A, Chowdhury D and Nishinari K 2010 Stochastic Transport in Complex Systems: from Molecules to Vehicles (Elsevier)

[42] Ben-Akiva M, Palma A D and Isam K 1991 Transp. Res. Part A: General 25251 $-266$

[43] für Verkehr des Landes Nordrhein-Westfalen M last access: May 2020 Verkehr nrw (traffic information for northrhine-westphalia) URL https://www.verkehr.nrw/

[44] Wahle J, Bazzan A L C, Klügl F and Schreckenberg M 2000 Physica A: Statistical Mechanics and its Applications 287669 - 681

[45] Wikipedia contributors 2018 Wikimedia Foundation Inc. (Online, accessed 10-August-2018) URL https://en.wikipedia.org/wiki/Advanced_traveller_ information_system

[46] comScore 2016 Statista - The Statistics Portal (Online, accessed 11Septmenber-2018) URL https://www.statista.com/statistics/250862/ unique-visitors-to-the-most-popular-mobile-apps-in-the-us/

[47] Barth D 2009 Google (Online, accessed 12-September-2018) URL https:// googleblog. blogspot. com/2009/08/bright-side-of-sitting-in-traffic. html 
[48] Brindle B 2014 HowStuffWorks (Online, accessed 10September-2018) URL https://electronics.howstuffworks.com/ how-does-google-maps-predict-traffic.htm

[49] Li B, Lokhov A Y and Saad D 2020 Reducing urban traffic congestion due to localized routing decisions (Preprint 2002.10298)

[50] Thai J, Laurent-Brouty N and Bayen A M 2016 19th International Conference on Intelligent Transportation Systems (ITSC) (IEEE) pp 595-601

[51] Horowitz J L 1984 Transp. Res. Part B: Methodological $1813-28$

[52] Hall R W 1996 Transp. Res. Part C: Emerging Technologies 4289 - 306

[53] Arnott R, de Palma A and Lindsey R 1991 Transp. Res. Part A: General 25309 $-318$

[54] Bazzan A L and Klügl F 2003 Proceedings of the first international workshop on Evolutionary Game Theory for Learning in MAS

[55] Bazzan A L C and Klügl F 2005 Transp. Res. Part C: Emerging Technologies 13 299-319

[56] Bazzan A L C, Wahle J and Klügl F 1999 Lect. Notes Comp. Sci. 1701 303-307

[57] Nagel K and Schreckenberg M 1992 Journal de Physique I 2 2221-2229

[58] Lee K, Hui P M, Wang B H and Johnson N F 2001 J. Phys. Soc. Jpn. 70 3507-3510

[59] Wang W X, Wang B H, Zheng W C, Yin C Y and Zhou T 2005 Phys. Rev. E 72(6) 066702

[60] Chen B K, Tong W, Zhang W Y, Sun X Y and Wang B H 2012 EPL (Europhysics Letters) 9714001

[61] He Z, Chen B, Jia N, Guan W, Lin B and Wang B 2014 Int. J. Mod. Phys. C 25 1450005

[62] Levy N and Ben-Elia E 2016 Procedia Computer Science 83 928-933

[63] Ben-Elia E and Avineri E 2015 Transport Reviews 35 352-377

[64] Ye H, Xiao F and Yang H 2017 Transp. Res. Procedia 23679 - 699

[65] Mak V, Seale D A, Gisches E J, Yang R, Cheng M, Moon M and Rapoport A 2018 Production and Operations Management 27 717-733

[66] Bittihn S 2018 Dissertation, University of Cologne URL https://kups.ub. uni-koeln.de/9166/ 